\title{
Standardized evaluation methodology and reference database for evaluating IVUS image segmentation
}

\author{
Simone Balocco ${ }^{\mathrm{a}, \mathrm{b}, *}$, Carlo Gatta $^{\mathrm{a}}$, Francesco Ciompi ${ }^{\mathrm{a}, \mathrm{b}}$, Andreas Wahle $^{\mathrm{c}}$, Petia Radeva ${ }^{\mathrm{a}, \mathrm{b}}$, \\ Stephane Carlier ${ }^{\mathrm{d}}$, Gozde Unal $^{\mathrm{e}}$, Elias Sanidas ${ }^{\mathrm{f}}$, Josepa Mauri ${ }^{\mathrm{g}}$, Xavier Carillo ${ }^{\mathrm{g}}$, \\ Tomas Kovarnik $^{\mathrm{h}}$, Ching-Wei Wang ${ }^{\mathrm{i}}$, Hsiang-Chou Chen ${ }^{\mathrm{i}}$, Themis P. Exarchos ${ }^{\mathrm{j}}$, \\ Dimitrios I. Fotiadis ${ }^{\mathrm{j}}$, François Destrempes ${ }^{\mathrm{k}}$, Guy Cloutier $^{\mathrm{k}, \mathrm{l}}$, Oriol Pujol ${ }^{\mathrm{b}}$, \\ Marina Alberti ${ }^{\mathrm{a}, \mathrm{b}}$, E. Gerardo Mendizabal-Ruiz ${ }^{\mathrm{m}}$, Mariano Rivera ${ }^{\mathrm{n}}$, Timur Aksoy ${ }^{\mathrm{e}}$, \\ Richard W. Downe ${ }^{c}$, Ioannis A. Kakadiaris ${ }^{\mathrm{m}}$ \\ a Computer Vision Center, Bellaterra, Spain \\ b Dept. Matemàtica Aplicada i Anàlisi, Universitat de Barcelona, Barcelona, Spain \\ ${ }^{c}$ Department of Electrical \&' Computer Engineering, The University of Iowa, Iowa City, USA \\ d UZ Brussel, Department of Cardiology, Brussels, Belgium \\ e Faculty of Engineering and Natural Sciences, Sabanci University, Turkey \\ ${ }^{\mathrm{f}}$ Cardiovascular Research Foundation, New York, USA \\ ${ }^{g}$ Hospital Universitari “Germans Trias i Pujol”, Badalona, Spain \\ h 2nd Department of Internal Medicine, Charles University, Prague, Czech Republic \\ ${ }^{\mathrm{i}}$ National Taiwan University of Science and Technology, Taiwan \\ j Institute of Molecular Biology and Biotechnology, Department of Biomedical Research, Foundation for Research and Technology Hellas, University of \\ Ioannina, Ioannina, Greece \\ ${ }^{\mathrm{k}}$ Laboratory of Biorheology and Medical Ultrasonics, University of Montreal Hospital Research Center (CRCHUM), Montreal, Canada \\ ${ }^{1}$ Department of Radiology, Radio-Oncology and Nuclear Medicine, Institute of Biomedical Engineering, University of Montreal, Montreal, Canada \\ m Computational Biomedicine Lab, Department of Computer Science, University of Houston, Houston, TX, USA \\ ${ }^{\mathrm{n}}$ Centro de Investigacion en Matematicas, Guanajuato, Mexico
}

\section{A R T I C L E I N F O}

\section{Article history:}

Received 27 September 2012

Received in revised form 15 March 2013

Accepted 1 July 2013

Keywords:

IVUS (intravascular ultrasound)

Evaluation framework

Algorithm comparison

Image segmentation

\begin{abstract}
A B S T R A C T
This paper describes an evaluation framework that allows a standardized and quantitative comparison of IVUS lumen and media segmentation algorithms. This framework has been introduced at the MICCAI 2011 Computing and Visualization for (Intra)Vascular Imaging (CVII) workshop, comparing the results of eight teams that participated.

We describe the available data-base comprising of multi-center, multi-vendor and multi-frequency IVUS datasets, their acquisition, the creation of the reference standard and the evaluation measures. The approaches address segmentation of the lumen, the media, or both borders; semi- or fully-automatic operation; and 2-D vs. 3-D methodology. Three performance measures for quantitative analysis have been proposed. The results of the evaluation indicate that segmentation of the vessel lumen and media is possible with an accuracy that is comparable to manual annotation when semi-automatic methods are used, as well as encouraging results can be obtained also in case of fully-automatic segmentation. The analysis performed in this paper also highlights the challenges in IVUS segmentation that remains to be solved.
\end{abstract}

(c) 2013 Elsevier Ltd. All rights reserved.

\footnotetext{
* Corresponding author at: Dept. Matemàtica Aplicada i Anàlisi, Universitat de Barcelona, Barcelona, Spain. Tel.: +34 934039053.

E-mail address: balocco.simone@gmail.com (S. Balocco).
}

\section{Introduction}

\subsection{Clinical background}

Cardiovascular diseases account for $30 \%$ of all deaths worldwide [1]. Atherosclerosis, a disease of the vessel wall, is the major cause of cardiovascular diseases such as heart attack or stroke [2].

Early atherosclerosis results in remodeling, thus retaining the lumen despite plaque accumulation [3]. In later stages, luminal 


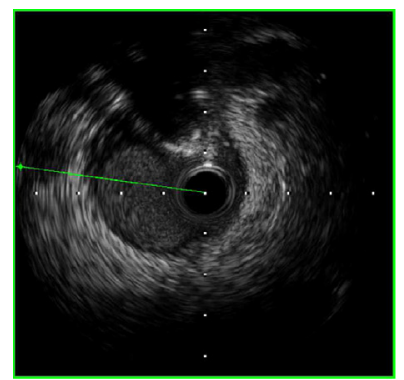

(a)

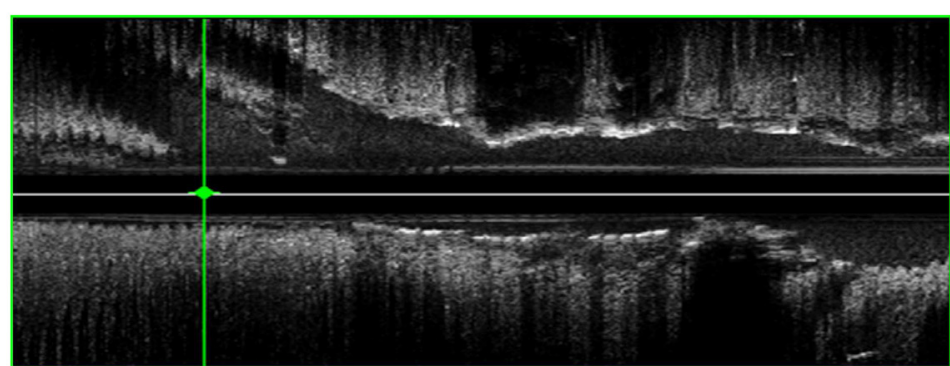

(b)

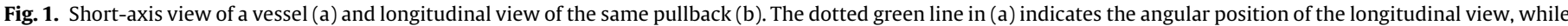

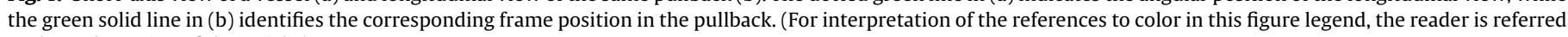
to the web version of the article.)

narrowing occurs, either diffuse over a vessel segment [4] or as localized stenosis. Only the latter case is usually directly visible using X-ray angiography. Intravascular Ultrasound (IVUS) allows monitoring and quantifying the state of the vessel wall and lumen. In addition, IVUS is an intra-operative imaging tool for the quantification and characterization of coronary plaque, used for diagnostic purposes and for guiding Percutaneous Coronary Intervention (PCI), enabling the visualization of high resolution images of internal vascular structures. Finally, IVUS is a fundamental tool for stent deployment because it allows assessment of the intervention and the correct placement of the device.

The acquisition of an IVUS sequence consists of inserting an ultrasound emitter, carried by a catheter, into the arterial vessel and pulling the probe from the distal to the proximal position (pullback). The standard IVUS image is a 360-degree tomographic cross-sectional view of the vessel walls, denoted as short-axis view (Fig. 1a), which allows an accurate assessment of vessel morphology. Given an angular position on the short-axis view (indicated in Fig. $1 \mathrm{~b}$ by a dotted line), the correspondinglongitudinal view can be generated by considering the gray-level values of the sequence along the diameter at the chosen angle. This longitudinal image depicts the morphology of the vessel section according to the selected orientation. Compared with other angiographic imaging modalities, (e.g. X-ray, MRA and CT), IVUS enables the visualization of both vessel morphology and plaque, and it provides extremely high image resolution (up to $113 \mu \mathrm{m}$ ). Such characteristics are essential for the clinical diagnosis since to date it is the only modality enabling the accurate morphological segmentation of both vessel membranes (lumen and media) and the assessment of the plaque type in vivo [5,6]. Additionally, when IVUS is fused with X-ray projections, three dimensional plaque quantification and reconstruction can be obtained [7,8] (Fig. 2).

Since a typical pullback contains more than 3000 IVUS frames, an accurate (semi-)automatic assessment of lumen and media contours is highly desirable to reduce the workload of the physician, and to speed up the diagnosis. In particular, the manual evaluation needs to be performed by expert physicians because the images are affected by speckle noise, and the textural appearance may significantly vary according to the echograph brand and by the type of probe used in the acquisition.

\subsection{State of the art}

Manual lumen and media segmentation is a laborious task that suffers from inter- and intra-observer variabilities due to the high amount of noise and artifacts present on the IVUS images. Consequently, much work has been performed on (semi-)automated IVUS image processing as illustrated by Katouzian in a recent review [9]. After a brief summary of the most recent lumen and media segmentation algorithms, we present a comparison of several segmentation algorithms performed using the proposed evaluation framework. It has to be noted that our state of the art description partially overlaps with the one presented in [9]; hence, the reader interested in an exhaustive review is referred to [9].

\subsubsection{Lumen segmentation}

Automated lumen segmentation of IVUS sequences has been a topic of interest since the early 1990s. Many of the early approaches were based on the use of local properties of the image such as pixel intensity and gradient information (edges) combined with computational methods including graph search $[10,11]$, active surfaces [12], active contours [13], and neural networks [14]. In later approaches, segmentation was accomplished by the use of gray level variances to model ultrasound speckle [15], contrast of regions [16], statistical properties of the image [17,18], spatiotemporal information (3D segmentation) [19], and discrete wavelet decomposition [20]. Recently, a shape-driven method for lumen and media-adventitia segmentation was introduced by Unal et al. [21]. In this work, the lumen and media-adventitia contours were constrained to a smooth, closed geometry. Then, a shape space was built using training data and principal component analysis (PCA). Finally, segmentation was performed on this shape space by minimizing an energy function using nonparametric probability densities with global measurements. Taki et al. [22] proposed a method for the identification of the vessel borders. This method consisted of a preprocessing step followed by the geometric deformation of parametric models using edge information. Downe et al. [23] introduced a method where principal component analysis was used for pre-processing, while active contour models were used

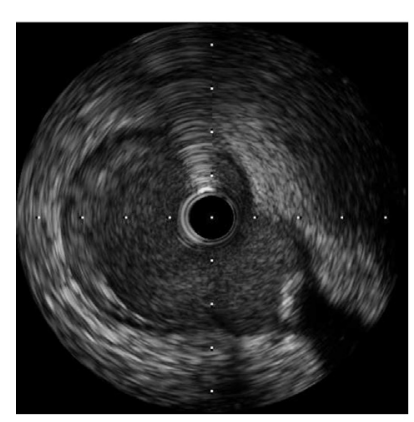

(a)

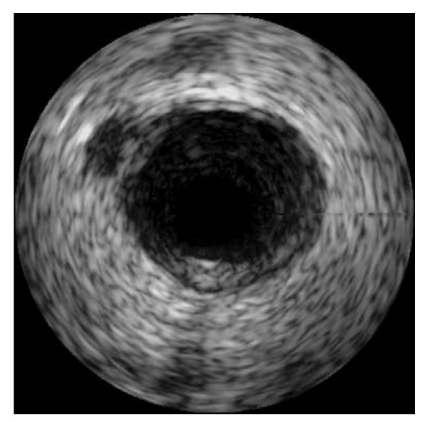

(b)
Fig. 2. IVUS Images from different systems acquired with a $40 \mathrm{MHz}$ (a) and $20 \mathrm{MHz}$ (b) probe. In the first image, two typical image artifacts are illustrated: the catheter guide produces a shadow artifact, and a calcium plaque hides the texture of the tissues lying behind it. The second image shows a vessel affected by a calcified plaque, and having a small branching vessel about to join the main artery. 
to provide an initial segmentation for a 3D graph search method. Multilevel discrete wavelet frames decomposition was used by Papadogiorgaki et al. [24] to generate texture information that was used along with the intensity information for contour initialization.

Similarly, Katouzian et al. [25] presented a method where texture information was extracted using a discrete wavelet packet transform. Then, pixels were classified as lumen or non-lumen using k-means clustering. Finally, the contour was parameterized using a spline curve. Mendizabal-Ruiz et al. [26] presented a probabilistic segmentation method based on the minimization of a cost function which deformed a contour parameterized represented by a one dimensional periodic function. In this method, the likelihood of each pixel to belong to lumen are computed using samples of the regions of interest on a number of frames of the sequence to be segmented. This method is capable of segmenting the lumen employing either the B-mode reconstruction images or the radio frequency (RF) IVUS data [27]. Ciompi et al. [28] presented a method in which segmentation was tackled as a classification problem and solved using an error correcting output code technique. In that work, contextual information was exploited by means of conditional random fields computed from training data. Wennogle and Hoff [29] proposed improvements over the method presented in [19] which include a preprocessing step to remove motion artifacts, a new directional gradient velocity term, and a post-processing level-set method. Roy Cardinal et al. [30] presented a multiple interface 3D fast-marching method that was based on a combination of gray level probability density functions and the intensity gradient. The segmentation method included an interactive initialization procedure of the external vessel wall border. Moraes et al. [31] proposed a method on which preprocessing and feature extraction over the IVUS images is performed, and then binary morphological object reconstruction is used to find the contours. Zhua et al. [32] presented a method based on the use of a linear-filtered gradient vector flow which drives the deformation of a balloon snake. Sun and Liu [33] presented a two step method which first detects the contours of interest on a number of L-mode cuts of the sequence and then evolve contours on the B-mode images until they reach the target points given by the first step. Finally, Balocco et al. [34] proposed an approach to automatically segment the vessel lumen, which combines model-based temporal information extracted from successive frames of the sequence, with spatial classification using the Growcut algorithm [35].

\subsubsection{Media segmentation}

As for the lumen border detection, several techniques have been proposed for the automatic and semi-automatic detection of the media border in IVUS. Most of the approaches rely on the idea that the most useful information for the media assessment are: (1) the local appearance of the vessel in proximity of the media layer and (2) the vessel shape.

Regarding the hypothesis on tissue appearance (1), the most exploited assumption relies on the echogenicity of media and adventitia tissues. The common pattern describing a gray level transition dark-bright in the media-adventitia interface has been used by several authors as initial approximation of the media. For this purpose, gradient-based operators as well as edge detectors have been used [11,14,36,21,22,37,31,38,24]. In some approaches some kind of user interaction is required $[39,11,14]$. Sonka et al. [11] proposed a method that uses knowledge on the local appearance of the media-adventitia interface while guiding a graph search. Papadogiorgaki et al. [24] employ an algorithm where the media-adventitia border is initialized based on gray level intensity. Additionally, techniques of median filtering [36,21], despeckle $[22,37,40]$ or edge detection by means of the Canny operator $[22,38]$ are used during border initialization.
The second common hypothesis (2) for media definition relies on the smoothness and continuity of the vessel shape. To this aim, given an initial approximation of the media, several approaches use a deformable model to compute the final vessel border $[41,14,18,36,42,22,37,19,43,12,30]$. The evolution of the model is commonly guided by an energy function, embedding information on the vessel morphology, gray level distribution [19,30], edge information by gradient [36,38,21,30], image intensity [42] and image contrast [41].

In some approaches, the information on the vessel shape and tissue properties is obtained by means of a learning process rather than using local per-image observation. Unal et al. [21] propose a strategy where the shape of the media is learned from training examples to create a shape space that is used to find the borders. Gil et al. [18] present an approach based on a learning task applied to tissue characteristics by defining the class calcification and vessel border and discriminated by a Fisher classifier. Mojsilovic et al. [44] propose an algorithm that describe plaque, lumen and adventitia regions by means of two textural features, and then classify them in an unsupervised fashion. An unsupervised classification is also proposed by Moraes and Furuie [40], where the Otsu thresholding [45] is applied to wavelet-based features to separate the adventitia from the plaque region.

Given the implicit ambiguity of the media appearance, the idea of reproducing the human reasoning while detecting the media has been exploited as well. Bovenkamp et al. [46] aim at encoding the relationships between the parts constituting the vessel morphology by means of a set of agents. Using a similar idea, a two-steps approach was presented by Olszewski et al. [47]. Regions that are most likely to belong to the media are first described at low resolution by means of an operator learned by training examples. Then, dynamic programming defines the final border at a higher resolution. In the algorithm of Mojsilovic et al. [44], a priori assumptions on vessel morphology are used to guide the media definition while refining classification results by morphological filters. Papagodiorgaki et al. [24] present a method that exploits only the relationship between lumen and media-adventitia border during initialization.

The ad hoc detection of regions hindering the vessel continuity, as calcifications or side-branches (bifurcations) has also been exploited. The detection of such artifacts allows, in some cases, to avoid an inaccurate border detection where the vessel appearance is partially occluded [21]. Plissiti et al. [14] makes use of a neural network based on a priori knowledge about the geometry of the vessel to overcome the effect due to calcifications. Dijkstra et al. [48], take profit of the presence of a stent manually detected by the user in order to define the lumen border within a framework that detects both lumen area and media in IVUS. Taki et al. [22], make use of a Bayesian classifier and a thresholding procedure on grey level to detect calcifications. Finally, in some approaches, the extension to 3D computation is proposed, or even the combination of cross-sectional and long-axis view [48], in order to improve the media detection $[12,19]$.

\subsection{Motivation}

As explicitly indicated in the review paper [9], direct performance comparisons of different approaches is difficult due to the lack of standard databases and validation criteria for most vascular segmentation applications. Moreover, many of the reviewed algorithms are not publicly available; this fact hampers an objective and fair comparison by third parties.

Nowadays, in medical imaging and computer vision communities, there is a growing number of initiatives that set up a publicly available data repository and standardized evaluation framework. This demonstrates an increasing interest in standardized evaluation and the possibility to compare methods to each other. In 
this section, we provide a few examples and we briefly discuss some published vascular segmentation algorithms. A standardized evaluation framework for carotid bifurcation lumen segmentation and stenosis grading, and for evaluating coronary artery centerline has been recently published $[49,50]$. Several workshops involving the setup of an evaluation framework in the field of medical imaging, have been organized at the MICCAI, ISBI and SPIE conferences. Reports on some of these frameworks have appeared recently [49,51-53]. More initiatives can be found at the website http://www.grand-challenge.org/.

The evaluation of lumen and media segmentation in IVUS has been introduced at the Computing and Visualization for (Intra)Vascular Imaging (CVII) workshop during the MICCAI 2011 conference held in Toronto [54,55], and this paper is an extended version of the challenge result evaluation presented at the workshop. Researchers interested in comparing the performance of new IVUS segmentation algorithms are encouraged to register on the site [56].

Finally the goals of this paper are (i) to suggest algorithms and databases for benchmarking, (ii) to propose three performance measures for quantitative analysis and (iii) to highlight the current challenges in IVUS segmentation.

\section{Evaluation framework}

In this section, a novel standardized evaluation methodology and reference database for evaluating IVUS image segmentation methods is presented.

\subsection{IVUS datasets}

The appearance of IVUS images can be extremely different depending on several factors which can be related to the system used in the acquisition (e.g. model, probe frequency, imaging artifacts, etc.), or to the morphology of the analyzed vessel (e.g. presence of bifurcation, plaques, shadows, proximity of the probe to the vessel, etc.). In order to evaluate the generalization capability of IVUS segmentation algorithms, the clinical recordings include heterogeneous data, covering most of those image categories. Due to the large amount of images composing a full IVUS pullback, a subset of significative frames has been extracted. However, care was taken in providing a sufficient number of consecutive frames in order to fulfill the requirement of most of the state-of-the-art algorithms.

\subsubsection{Transducer frequency and model}

The IVUS images acquired with different commercial systems are affected mainly by two factors: the acquisition hardware system and the use of customized transducer working at specific central frequencies. In order to evaluate the flexibility of the segmentation algorithm on different IVUS image appearance, two commercial echographs commonly used in clinical practice are considered. Each system was equipped with a different probe working at $20 \mathrm{MHz}$ and $40 \mathrm{MHz}$ central frequency, respectively. The images were divided in two datasets:

- Dataset A: A set of 77 images extracted from in vivo pullbacks of human coronary arteries, from 22 patients. The Imaging System used for the acquisition is an iLab IVUS (Boston Scientific, Fremont), equipped with a $40 \mathrm{MHz}$ catheter Atlantis SR 40 Pro.

- Dataset B: A set of 435 images extracted from in vivo pullbacks of human coronary arteries, from 10 patients. The imaging system used for the acquisition is a Si5 (Volcano Corporation), equipped with a $20 \mathrm{MHz}$ Eagle Eye monorail catheter.

\subsubsection{Dataset organization}

IVUS segmentation methods may exploit the IVUS pullback context using different strategies. Some approaches are based on the analysis of a single short-axis frame, others may require the full 3D volumetric pullback gated at the same cardiac cycle, some others take advantage of the information of consecutive frames in order to exploit the speckle correlation or the redundancy of the texture. For these reasons, the datasets are designed in such way to allow different approaches. Additionally, dataset A contains both gray level DICOM images and "Radio Frequency" (RF) raw data, allowing the algorithm based on raw Ultrasound signal to exploit the richer information contained in the data.

- Dataset A: Single-frame dataset, in which the frames to be segmented are not consecutive and are chosen at random instants of the cardiac cycle (not gated). For each frame, four adjacent images (two previous and two successive) to the extracted frame in DICOM \& RF formats are also provided.

- Dataset B: Multi-frame datasets, in which 3D context from a full pullback is provided. Between 20 and 50 gated frames are extracted from the full pullback at the end-diastolic cardiac phase. For each frame, four adjacent images in DICOM format are also provided.

The dataset B has been designed so to provide a sufficient number of frames for a volumetric analysis of the pullback. Indeed, IVUS pullbacks are characterized by a swinging effect (or artifact) due to the cardiac contraction. Such artefact provokes transversal oscillation of the catheter (axially, forth and back the vessel), which generates multiple sampling of the same spatial position of the vessel [57-59]. In order to obtain a coherent spatial scan of the vessel (a 3D topographic reconstruction of its morphology) the pullback frames belonging to a specific cardiac phase should be extracted (by ECG gating or image-based gating). Any other frame in the pullback corresponds to a different cardiac phase, hence the artery diameter might have expanded, and the position of the IVUS frame is unknown along the vessel morphology. As a consequence of the swinging, if we consider a specific frame F, the IVUS frame that spatially proceeds $\mathrm{F}$ (i.e. lies before $\mathrm{F}$ in the vessel) is not its adjacent, but it is the previous gated frame (which for instance may lie around 30 frames before the frame F). Considering this fact, a volumetric assessment of the pullback should be performed considering the gated frames only (the ones which have been manually annotated). Likewise, the frames that provide spatially relevant context for the segmentation of a given frame are the previous and following gated frames and not the adjacent frames. However, it must be noticed that some algorithms might need frames that are adjacent in the pullback acquisition (i.e. are temporally adjacent) in order to exploit the de-correlation of the blood scatterer as feature for the lumen segmentation. For this reason, an empirical number of five temporally adjacentframes around the frame $\mathrm{F}$ (two before and two after) have been included. It has been considered when more than two frames forward (and backward) in time are considered, consecutive IVUS frames might not be aligned. This is due to the rotation artifacts typical of an IVUS pullback [57-59], and because the vessel might start pulsating, and hence changing its diameter. Due to these two phenomena the correlation between successive frames could not be exploited by the algorithms. The capability of an algorithm to segment a frame extracted from a generic cardiac phase has been evaluated using dataset $A$. In the previous IVUS segmentation challenge only the IVUS frames strictly needed for the competition were provided. However the availability of a full pullback might be included in future IVUS competitions in order to include methods requiring the whole sequence. 


\subsubsection{Training and test sets}

Each of the two datasets (A and B) is divided into two groups (training and test sets) with the aim to assure the blindness of the algorithm evaluation with respect to the reference manual annotations. Such subsets are defined as follows:

- A training set: A subset (one fourth) of images from each dataset containing both images and manual annotations. The training set is meant for training and tuning of the algorithms prior to the evaluation. This dataset is distributed upon request for algorithm evaluation.

- A test set: The remaining frames compose the set of images that are used for performance evaluation. The annotations are not available before the algorithm evaluation in order to assure the complete blindness and equity of the algorithm assessment.

A Matlab (MathWorks ${ }^{\odot}$, Inc.) script completes the training set in order to provide a transparent mechanism to automatically evaluate the algorithm performance.

For segmentation methods that require manual initialization, such an interaction has been allowed as long as a detailed description was submitted together with the algorithm description. No manual editing of the algorithm results is allowed.

\subsubsection{Gold standard}

Annotations on the image datasets have been provided by four clinical experts, daily working with the specific IVUS echograph brand, and belonging to distinct medical centers. The annotation consists in the delineation of both inner wall (lumen contours) and outer wall (media/adventitia contours) on the IVUS images in the short-axis view. In all the cases, the experts were blinded to the other physicians' annotations, and two of them repeated the manual annotations after about one week from the first labeling.

\subsubsection{Image segmentation categories}

In an IVUS pullback not all the images have the same segmentation complexity, since echographic reflexions, probe artifacts, plaque appearance may make the segmentation task more challenging. For this reason, it is important to identify whether an algorithm is robust enough to segment all the frames or a good performance is obtained only in a specific image category.

For this purpose, the datasets have been labeled according to their morphological content and separated according to the categories illustrated in Table 1. An exemplar image of each category has been included in Table 1.

It is important to note that, with respect to the dataset B, the Volcano catheter slides over the guide-wire, thus it does not appear in the images. Additionally, in the particular vessel segments imaged, no angioplasty was performed during the session. For these reasons, no frames were assigned to three of the seven categories (Table 1).

Table 2 illustrates general statistics about the arteries included in the datasets. The average and standard deviation measures of lumen and media areas and stenosis degree, respectively are reported. The stenosis degree is defined as the ratio between media and lumen in an IVUS frame.

\subsubsection{Evaluation measures}

Three evaluation measures are chosen for each contour $C$. The Jaccard measure quantifies how much the segmented area overlaps with the manual delineated area; the PAD computes the segmentation area difference, and it is a general measurement used in clinical environments; the Hausdorff Distance computes locally the distance between the manual to the reference contour. Such measures are defined as follows:

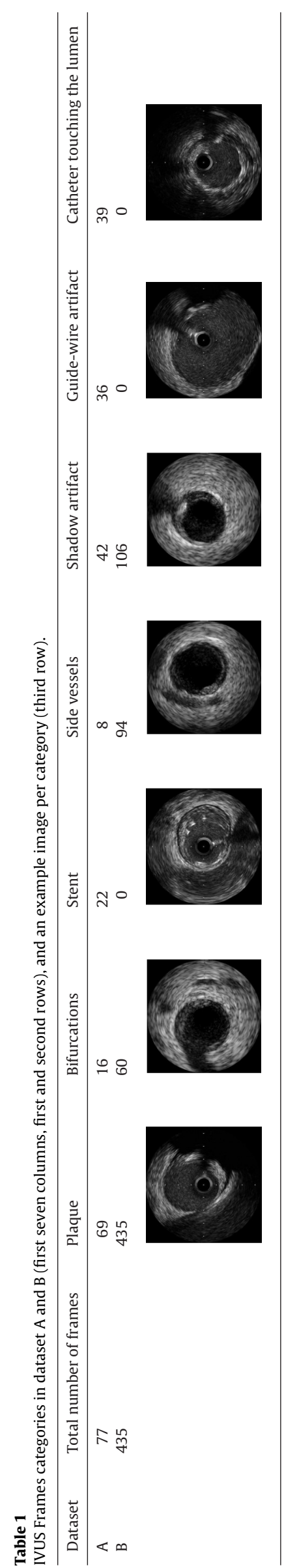


Table 2

Statistics of the arteries included in the datasets. The average and standard deviation measures of lumen and media areas and stenosis degree, respectively is reported.

\begin{tabular}{lll}
\hline Dataset & A & B \\
\hline Lumen area [mean (std)] & $10.62(5.62) \mathrm{mm}^{2}$ & $8.68(3.23) \mathrm{mm}^{2}$ \\
Media area [mean (std)] & $18.21(6.70) \mathrm{mm}^{2}$ & $15.59(4.47) \mathrm{mm}^{2}$ \\
Stenosis degree [mean (std)] & $57 \%(14 \%)$ & $54 \%(9 \%)$ \\
\hline
\end{tabular}

The Jaccard measure (JM) is computed over the two vessel areas defined by the automatic and the manual curve:

$J M\left(R_{\text {auto }}, R_{\text {man }}\right)=\frac{\left|R_{\text {auto }} \bigcap R_{\text {man }}\right|}{\left|R_{\text {auto }} \bigcup R_{\text {man }}\right|}$

where $R_{\text {auto }}$ and $R_{\text {man }}$ are two vessel regions defined by the manually annotated contours $C_{\text {man }}$ and of the (semi)automatic segmented outline $C_{\text {auto }}$, respectively.

Percentage of Area Difference (PAD) is the difference between the vessel area for the automatic $\left(A_{\text {auto }}\right)$ and manual $\left(A_{\operatorname{man}}\right)$ border expressed as a measure relative to the manual annotation:

$P A D=\frac{\left|A_{\text {auto }}-A_{\text {man }}\right|}{A_{\text {man }}}$

Hausdorff Distance (HD) between the automatic and the manual curve annotation, is computed as follows:

$H D\left(C_{\text {auto }}, C_{\text {man }}\right)=\max _{a \in C_{\text {auto }}}\left\{\max _{b \in C_{\operatorname{man}}}[d(a, b)]\right\}$

where $a$ and $b$ are points of the curves $C_{a u t o}$, and $C_{\text {man }}$, respectively, and $d(a, b)$ is the Euclidean distance.

\subsection{Methods for segmenting IVUS images}

The evaluation framework has been initially made available during the IVUS challenge, held at the MICCAI Workshop on Computing and Visualization for (Intra)Vascular Imaging in Toronto on September 18th, 2011.

In the competition, eight algorithms (labeled from P1 to P8), were evaluated and compared using the framework. Four algorithms were fully automatic, four were semi-automatic (i.e. required a manual initialization provided by an expert). Four methods were used to segment both lumen and media contours, while others were specifically designed for assessing the contours of one of the two. Table 3 summarizes the laboratories involved in the competition, while Table 4 provides an overview of each method's features, including whether the algorithm is applied to lumen and/or media, which dataset has been used, and the time required for the computing a frame.

\subsection{Method description}

The competition was open to any IVUS segmentation algorithm, hence published and unpublished approaches were allowed to compete. In this section, the key features of each method are briefly summarized, and a more detailed description of the approach is provided for unpublished algorithms.

\subsubsection{Participant 1 - Lumen and media}

This method [21] segments lumen and media layers in arterial walls by a linear projection of the inner (lumen) and outer (media) contour space onto two low dimensional prior shape spaces for each border. The algorithm runs on the rectangular (not-scan converted) IVUS image domain. A lumen contour is initialized by shifting the template (average) shape of the prior lumen shape space according to the intensity average above the average shape.
The lumen contour is evolved by an Euler-Lagrange equation based on the probabilities of intensities inside and outside the lumen $P_{\text {in }}$ and $P_{\text {out }}$, computed from a Parzen window that is estimated by histogram of intensities:

$\frac{\partial w_{i}^{l}}{\partial t}=\int_{C}-\log \left(\frac{P_{\text {in }}(I(x))}{P_{\text {out }}(I(x))}\right) U_{i}^{l} d x$

where $U_{i}^{l}$ is the eigenshape corresponding to the $i$ th lumen contour weight $w_{i}^{l}$.

A media contour is initialized by finding the maximum of smoothed gradients at regular intervals of the rectangular image, and evolved by a speed which is proportional to the gradient difference of two oriented windows above and below the contour $\nabla G$.

$$
\frac{\partial w_{i}^{a}}{\partial t}=\int_{C} \nabla G(x) U_{i}^{a} d x
$$

where $U_{i}^{a}$ is the eigenshape corresponding to the $i$ th media contour weight $w_{i}^{m}$.

Calcifications and openings due to side branches are detected before segmentation of each IVUS pullback frame, as features to be used in segmentations of lumen and media borders.

\subsubsection{Participant 2 - Lumen}

The segmentation method includes a smart blur function based on a median filter to reduce variations of pixels in the lumen areas, a hole filling step that replaces the IVUS catheter and the white dots with the texture of the neighboring data, and a geodesic active contour algorithm [60] for image segmentation. The active contour method does not perform well on the DICOM images because of the typical vertical and horizontal dots used as ruler and IVUS catheter ring-down artifact. These factors forbid active contour algorithms to grow properly and segment the lumen regions accurately. Therefore, a hole filling process is conducted to flatten these areas. The IVUS catheter is filled with the mean value of its outbound neighboring circular area. Then, as the images are in polar coordinates, the eight vertical white dots are replaced with the contents of the right-hand neighboring rectangles and the eight horizontal white dots are filled in with the contents of the neighboring rectangles below. After the hole filling process, images of both data sets are processed with a "smart blur" algorithm to further smoothen the information in order to make local feature more relatively consistent. The "smart blur" method preferentially blurs parts of an image that are sparse in detail (rich in low-frequency information) while leaving untouched the parts of the image that are comparatively rich in detail (rich in high-frequency information). In other words, abrupt transitions in tone are ignored; areas of subtle change are smoothed (and thus made even more subtle). Next, the geodesic active contour algorithm is applied with manual initialization. Coordinates of the output contour are then extracted to a text file for evaluation.

\subsubsection{Participant 3 - Lumen and media}

The method, which has been tested on the 20 and $40 \mathrm{MHz}$ data sets, represents an improvement of the technique initially developed for 3D ungated pullbacks acquired at $20 \mathrm{MHz}[30,19]$. After initialization, images were represented in the polar domain. A mixture model of four gamma statistical distributions was adopted for the gray levels, and estimated with the Expectation-Maximization algorithm on regions based on translations of the initial boundaries. For each tissue representing: (i) the guide wire and the lumen, (ii) the intima, (iii) the media and (iv) the surrounding tissues, respectively, as well as any other artifact present within the image, the weight of each distribution for each tissue was estimated on the 
Table 3

Participants to the challenge competition held at the MICCAI 2011 Computing and Visualization for (Intra)Vascular Imaging (CVII) workshop.

\begin{tabular}{|c|c|c|c|}
\hline Label & Institution & First author & City, Country \\
\hline P1 & Sabanci University & Timur Aksoy & Istanbul, Turkey \\
\hline P2 & National Taiwan University of Science and Technology & Ching-Wei Wang & Taipei, Taiwan \\
\hline P3 & University of Montreal & François Destrempes & Montreal, Canada \\
\hline P4 & University of Iowa & Richard W. Downe & Iowa City, USA \\
\hline P5 & Universitat de Barcelona & Marina Alberti & Barcelona, Spain \\
\hline P6 & Universitat de Barcelona & Francesco Ciompi & Barcelona, Spain \\
\hline P7 & University of Houston & Gerardo Mendizabal-Ruiz & Houston, USA \\
\hline P8 & Biomedical Research Institute & Themis P. Exarchos & Ioannina, Greece \\
\hline
\end{tabular}

same regions. The initialization process was adapted to the temporal 2D sequences, and only their first frames were considered for the Probability Density Functions (PDFs) estimation. The FastMarching Method (FMM) speed function combined gradient-based contour information and a textural gradient, based on the estimated gray level PDFs of the vessel structures, which took into account the great variability in appearance of the tissues and the lumen in IVUS images (especially at $40 \mathrm{MHz}$ ). The two gradients were normalized so that their average values were equal on the initial FMM far-away region of each image. A Gaussian filter was applied to the segmented boundaries. The external elastic membrane and then the lumen were segmented and the boundaries were then converted into the Cartesian domain.

\subsubsection{Participant 4 - Lumen and media}

This method [23] segments the coronary arteries by first computing a cost function for each of the two borders, and then using the graph search method outlined in [61] to solve the optimal surface detection problem based upon the cost function. The cost function is computed separately for each border, and the graph search simultaneously minimizes the cost of both in search of an optimal solution.

The inner border cost function uses an active contour estimate driven by proximity to an intensity threshold to detect the inner border. The threshold is computed using the method of [45]. The cost terms are based upon exponential distance from the estimate, along with intensity based regional cost information in close proximity to the estimate. These regional cost terms are based upon the notion that a positive gradient should exist moving outward from the border, and an absence thereof is penalized. The outer border costs are determined using a $7 \times 7$ 3-d edge detection mask. The edge convolution is performed on a log-polar image, and wrapped at the ends, which improves the image edge conformance to a rectangular shape.

This algorithm requires no initialization. However, the center from which the log-polar representation is computed, and from which the active contour estimate is grown, can be adjusted prior to the segmentation if the image is significantly out of the center in the frame. Any catheter artifact must also be masked by dragging a circular cursor to enlarge it to cover the artifact, but this is necessary only in data-sets where such an artifact is present.

\subsubsection{Participant 5 - Lumen}

A binary classification problem is defined, aiming at distinguishing between lumen and non-lumen regions. A set of textural features are extracted from the polar IVUS image: Gabor filters, shade, relative shade and Cross-correlation [34]. The Multi-scale Stacked Sequential Learning (MSSL) scheme [62] is applied, as a way of capturing and exploiting spatial sequential label relationships, extended over multiple spatial scales. The originally bi-dimensional MSSL approach is extended to three dimensions, (i.e. the radial, angular and longitudinal dimensions of the IVUS sequence). In the first MSSL stage, each pixel of the IVUS image is independently classified, while in the second classification stage, the spatial neighborhood relation among pixels is exploited. In both phases, AdaBoost classifier with decision stumps is used.

The luminal border is then identified by applying an active contour to the obtained binary classification map. The external energy is computed as the first derivative of the classification map, previously blurred by Gaussian filtering, in both vertical and horizontal dimensions. Vertical derivatives of non-lumen to lumen transitions are discarded, since they do not represent lumen borders. The fitting of a snake is performed twice: a rough detection is first achieved by initializing the snake horizontally, while in a successive refinement, the Gaussian standard deviation is halved and the snake is initialized to the position reached in the previous stage.

\subsubsection{Participant 6 - Media}

The used method was presented in [63], where a robust border detection is achieved by means of a holistic interpretation of the detection problem where the target object (i.e. the media layer) is considered as part of the whole vessel in the image and all the relationships between tissues are learned. A fairly general framework exploiting multi-class tissue characterization as well as contextual information on the morphology and the appearance of the tissues is has been proposed.

The used method, namely HoliMAb, was presented in [63] as a fully automatic methodology for the detection of the MediaAdventitia border (MAb). The detection of the MAb is achieved by means of a holistic interpretation of the data domain (the IVUS image) where the target object (i.e. the media layer) is considered as part of the whole vessel in the image and all the relationships between tissues are learned. The algorithm is based on two steps.

Table 4

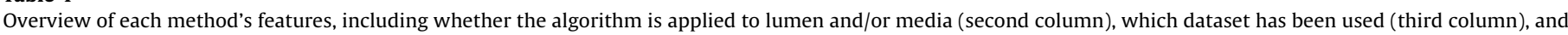
the time required for the computing a frame (forth and fifth columns).

\begin{tabular}{|c|c|c|c|c|c|c|}
\hline Label & Category & Dataset & Fully-Autom/Semi-Autom & $2 \mathrm{D} / 3 \mathrm{D}$ & Time per frame & Hardware used \\
\hline P1 & Lumen and media & B & SEMI & $2 \mathrm{D}$ & $3.25 \mathrm{~s}$ & Pentium $62002.13 \mathrm{GHz}$ \\
\hline P2 & Lumen & $A, B$ & SEMI & $2 \mathrm{D}$ & $1 \mathrm{~m} 40 \mathrm{~s}$ & Xeon $2.67 \mathrm{GHz}$ \\
\hline P3 & Lumen and media & $A, B$ & SEMI & $2 \mathrm{D}$ & $12.28 \mathrm{~s}$ (dataset A) $8.64 \mathrm{~s}$ (dataset B) & Core i7 Q740@1.73 GHz \\
\hline P4 & Lumen and media & B & AUTO & $3 \mathrm{D}$ & $0.16 \mathrm{~s}$ & Core $2,2.4 \mathrm{GHz}$ \\
\hline P5 & Lumen & $A, B$ & AUTO & $3 \mathrm{D}$ & $13 \mathrm{~s}$ & Core 2 Duo $2.13 \mathrm{GHz}$ \\
\hline P6 & Media & $A, B$ & AUTO & $2 \mathrm{D}$ & $20 s$ & Core i7 $2.8 \mathrm{GHz}$ \\
\hline P7 & Lumen & $A, B$ & SEMI & $2 \mathrm{D}$ & $16.95 \mathrm{~s}$ (dataset A) $4.96 \mathrm{~s}$ (dataset B) & Core i7 a $2 \mathrm{GHz}$ \\
\hline P8 & Lumen and media & $A, B$ & AUTO & $2 \mathrm{D}$ & $0.5 \mathrm{~s}$ & Core 2 Duo $3.33 \mathrm{GHz}$ \\
\hline
\end{tabular}


First, the vessel morphology is interpreted by means of a multiclass classification approach, where the eight main regions in the vessel are automatically labelled (blood, plaque, guide-wire, calcification, shadow, external tissue, media layer, adventitia tissue). For this purpose, authors demonstrate that using a context-aware classifier allows to obtain highly accurate tissue modeling, dealing with artifacts and noise in ultrasound. Secondly, a functional that expresses the relationship between the media-adventitia curve and the whole vessel is learned and used to guide the evolution of the MAb. In order to obtain the solution, the curve is always initialized as circle at half the maximum radial distance, and then its evolution is controlled by adding harmonics component according to the Fourier series of the curve function.

\subsubsection{Participant 7 - Lumen}

The IVUS images to be segmented are first converted into polar representation on which the lumen contour is parameterized using one-dimensional Fourier series. The lumen contour is deformed by the minimization of a cost function that is formulated using a Bayesian approach [26] in which the posterior probabilities are obtained employing level five Law's texture features and the probability estimates provided by a Support Vector Machine classifier with a radial basis function kernel build for the specific IVUS sequence to segment. The minimization of the cost function is performed by employing an ad-hoc optimization strategy in which the direction of the steepest descent and Broyden-Fletcher-Goldfarb-Shanno optimization methods are linearly combined to improve convergence. The use of a specific support vector machine classifier for each sequence to be segmented makes the method capable of segmenting IVUS B-mode images from different transducer frequencies (i.e. 20 and $40 \mathrm{MHz}$ ) without the need of any parameter tuning, and makes it robust with respect to different B-mode reconstruction parameters.

\subsubsection{Participant 8 - Lumen and media}

The algorithm for lumen and media-adventitia border detection is based on an improvement of the previously published algorithm [64]. The rationale is based on a sequential algorithm, which computes the two borders of interest in an IVUS frame using as initial information the two borders of the previous IVUS frame. The first frame of the IVUS is specially processed, in order to acquire the 2D borders which will be used as input to the sequential detection. The main novelty is on the automatic detection of the borders in the first IVUS frame. Initially, the first IVUS frame is transformed to a polar coordinate image. Then, the artifacts induced by the transducer are removed given its diameter. In order to remove artifacts due to the blood the filters are applied to the polar image. Then a Gaussian low pass filter is applied to the binary image to compute edges. Finally, for each angle the edge of minimum radius and the lumen border is considered. The media-adventitia border is represented by a thick bright zone in polar coordinates. Thus, the border for each angle is defined as the pixel of minimum radius with brightness value above an image adaptive threshold. In order to eliminate discontinuities on the border contours, the primary results by an averaging filter was then refined. Finally, the refined estimations are transformed back to Cartesian coordinates.

\subsection{Method initialization}

IVUS segmentation algorithms could require initialization strategies suitable for IVUS modality, which are different than those used in other imaging modalities. With the aim of allowing all algorithms to compete fairly with the best possible approach, arbitrary manual initializations were allowed. However, more complex initializations may lead to time-consuming procedures and tedious workload for the users. To take this fact into account,

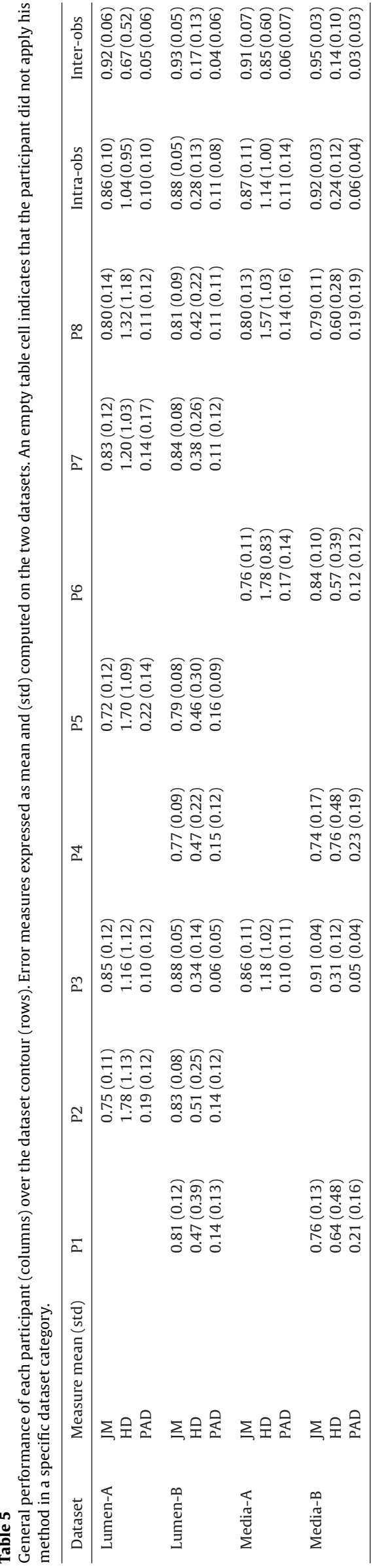

Please cite this article in press as: Balocco S, et al. Standardized evaluation methodology and reference database for evaluating IVUS image segmentation. Comput Med Imaging Graph (2013), http://dx.doi.org/10.1016/j.compmedimag.2013.07.001 
all semi-automatic methods were required to provide a detailed description of the initialization stage along with the segmentation results. In all cases, no manual edits to the results of the algorithm were allowed. Participants 4, 5, 6 and 8 developed fully-automatic methods whereas the methods requiring manual interaction were initialized as follows:

\subsubsection{Participant 1}

The algorithm initialization requires the manual definition of a Parzen window, which estimates the histogram of intensities in and outside of the lumen contour for the evolution of the curve. It is noted that initialization is in terms of an intensity distribution sampling rather than a geometric contour input.

\subsubsection{Participant 2}

The lumen initialization, performed by an expert user, consists in placing a starting box in the middle of the IVUS image. The active contour method propagates from the initial seed.

\subsubsection{Participant 3}

For the Data Set A: In the first Cartesian image of the temporal $2 \mathrm{D}$ sequence, four to eight points were selected on the two borders that were spline-interpolated to yield approximate initial boundaries. The video of the temporal sequence (in the Cartesian domain) of the five DICOM images was also available while doing the manual initialization for additional visualization (but no temporal information was used in the algorithm). For the Data Set B: On two longitudinal-views and for each boundary, three to eight points were selected and interpolated to provide four border points for each image, which after radial spline-interpolation yielded the initial boundaries. The polar images for a given position in the pullback were available.

\subsubsection{Participant 7}

Initialization requires an expert user to perform annotations of the lumen and non-lumen regions (i.e. plaque, vessel wall and adventitia) in the first Cartesian B-mode frame of the sequence to be segmented. It is required that the samples from lumen cover at least $50 \%$ of the total lumen area and that the annotations from non-lumen regions are selected in the areas close to the lumen border.

\section{Results evaluation}

The IVUS evaluation framework allowed a detailed and extensive comparison of the methods, illustrating the strength and the weaknesses of each approach. Quantitative results are reported in several numerical tables. In order to ease the analysis, several images provide a visual comparison of the approaches. Finally, a qualitative assessment of the results can be appreciated by analyzing the segmentation results on several exemplar frames extracted from the datasets $\mathrm{A}$ and $\mathrm{B}$.

\subsection{Quantitative evaluation}

For each method, the performance has been assessed by computing the evaluation measures described in Section 2.1.6. For each algorithm, the scores were obtained by comparing the contours provided by the participant against the manual observer annotations and listed in Tables 5-12. Additionally inter- and intraobserver variability was included in order to provide reference performance.

Specifically, Table 5 reports the overall performance (mean and standard deviation) of the method separated according to each dataset and each segmentation class (lumen and media). Then,

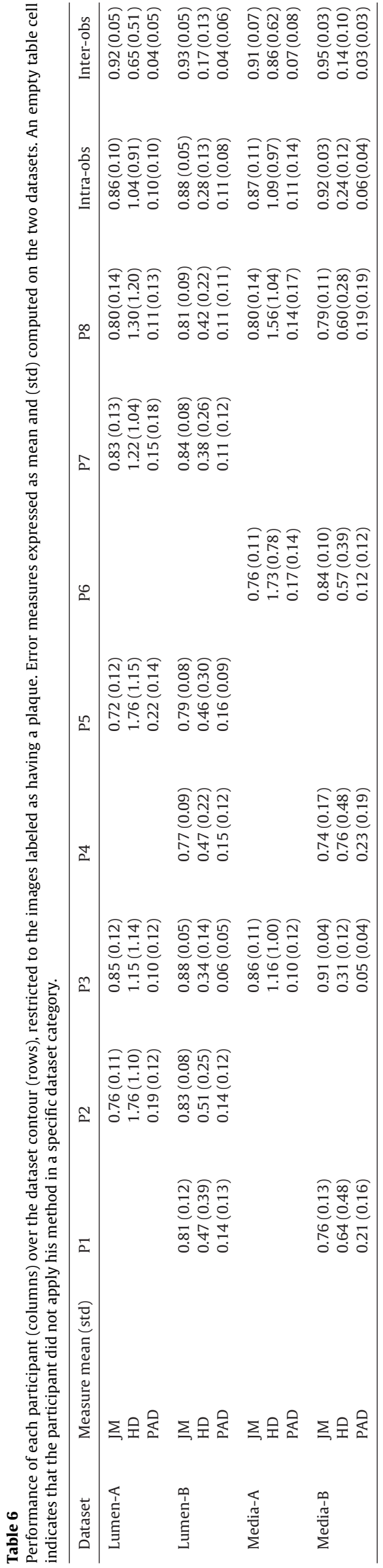

Please cite this article in press as: Balocco S, et al. Standardized evaluation methodology and reference database for evaluating IVUS image segmentation. Comput Med Imaging Graph (2013), http://dx.doi.org/10.1016/j.compmedimag.2013.07.001 
Table 7

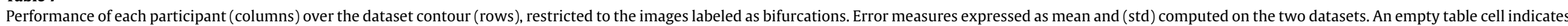
that the participant did not apply his method in a specific dataset category.

\begin{tabular}{|c|c|c|c|c|c|c|c|c|c|c|c|}
\hline Dataset & Measure mean (std) & P1 & P2 & P3 & P4 & P5 & P6 & P7 & P8 & Intra-obs & Inter-obs \\
\hline \multirow[t]{3}{*}{ Lumen-A } & JM & & $0.77(0.10)$ & $0.84(0.11)$ & & $0.72(0.09)$ & & $0.83(0.11)$ & $0.79(0.12)$ & $0.88(0.08)$ & $0.91(0.05)$ \\
\hline & HD & & $1.77(1.30)$ & $1.37(1.49)$ & & $1.66(1.30)$ & & $1.11(0.94)$ & $1.43(1.42)$ & $0.88(0.80)$ & $0.73(0.64)$ \\
\hline & PAD & & $0.19(0.09)$ & $0.09(0.10)$ & & $0.22(0.13)$ & & $0.14(0.18)$ & $0.13(0.12)$ & $0.05(0.04)$ & $0.05(0.05)$ \\
\hline \multirow[t]{3}{*}{ Lumen-B } & JM & $0.76(0.14)$ & $0.81(0.11)$ & $0.85(0.06)$ & $0.70(0.11)$ & $0.75(0.10)$ & & $0.79(0.12)$ & $0.80(0.09)$ & $0.88(0.04)$ & $0.92(0.07)$ \\
\hline & HD & $0.65(0.47)$ & $0.54(0.27)$ & $0.42(0.18)$ & $0.64(0.27)$ & $0.61(0.43)$ & & $0.53(0.36)$ & $0.47(0.23)$ & $0.30(0.12)$ & $0.18(0.21)$ \\
\hline & PAD & $0.18(0.15)$ & $0.14(0.13)$ & $0.08(0.06)$ & $0.21(0.15)$ & $0.20(0.10)$ & & $0.17(0.18)$ & $0.10(0.09)$ & $0.09(0.06)$ & $0.05(0.09)$ \\
\hline \multirow[t]{3}{*}{ Media-A } & JM & & & $0.81(0.14)$ & & & $0.75(0.11)$ & & $0.78(0.13)$ & $0.86(0.12)$ & $0.89(0.06)$ \\
\hline & $\mathrm{HD}$ & & & $1.56(1.36)$ & & & $1.68(0.84)$ & & $1.75(1.12)$ & $1.34(1.31)$ & $1.08(0.62)$ \\
\hline & PAD & & & $0.16(0.13)$ & & & $0.21(0.18)$ & & $0.17(0.20)$ & $0.11(0.12)$ & $0.08(0.07)$ \\
\hline \multirow[t]{3}{*}{ Media-B } & JM & $0.78(0.13)$ & & $0.91(0.03)$ & $0.71(0.19)$ & & $0.85(0.07)$ & & $0.78(0.11)$ & $0.92(0.02)$ & $0.95(0.04)$ \\
\hline & HD & $0.57(0.46)$ & & $0.32(0.13)$ & $0.79(0.53)$ & & $0.52(0.29)$ & & $0.63(0.25)$ & $0.24(0.09)$ & $0.15(0.13)$ \\
\hline & PAD & $0.19(0.15)$ & & $0.06(0.04)$ & $0.24(0.21)$ & & $0.09(0.07)$ & & $0.23(0.23)$ & $0.06(0.03)$ & $0.03(0.03)$ \\
\hline
\end{tabular}

Table 8

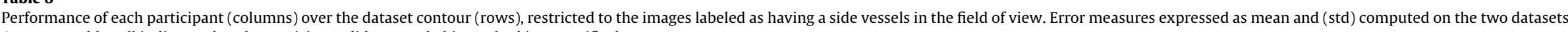
An empty table cell indicates that the participant did not apply his method in a specific dataset category.

\begin{tabular}{|c|c|c|c|c|c|c|c|c|c|c|c|}
\hline Dataset & Measure mean (std) & P1 & P2 & P3 & P4 & P5 & P6 & P7 & P8 & Intra-obs & Inter-obs \\
\hline \multirow[t]{3}{*}{ Lumen-A } & JM & & $0.78(0.04)$ & $0.84(0.13)$ & & $0.72(0.10)$ & & $0.84(0.10)$ & $0.78(0.16)$ & $0.82(0.09)$ & $0.92(0.06)$ \\
\hline & HD & & $1.49(0.32)$ & $1.07(0.74)$ & & $1.58(0.64)$ & & $0.92(0.61)$ & $1.28(0.95)$ & $1.22(0.75)$ & $0.55(0.40)$ \\
\hline & PAD & & $0.18(0.08)$ & $0.12(0.14)$ & & $0.18(0.11)$ & & $0.10(0.10)$ & $0.10(0.11)$ & $0.14(0.10)$ & $0.04(0.05)$ \\
\hline \multirow[t]{3}{*}{ Lumen-B } & JM & $0.79(0.12)$ & $0.80(0.10)$ & $0.87(0.04)$ & $0.77(0.08)$ & $0.79(0.07)$ & & $0.84(0.07)$ & $0.77(0.09)$ & $0.88(0.05)$ & $0.91(0.05)$ \\
\hline & HD & $0.51(0.39)$ & $0.59(0.23)$ & $0.36(0.15)$ & $0.46(0.19)$ & $0.47(0.24)$ & & $0.38(0.19)$ & $0.53(0.24)$ & $0.30(0.13)$ & $0.20(0.11)$ \\
\hline & PAD & $0.17(0.14)$ & $0.16(0.13)$ & $0.07(0.04)$ & $0.15(0.11)$ & $0.17(0.09)$ & & $0.11(0.11)$ & $0.16(0.12)$ & $0.10(0.08)$ & $0.06(0.05)$ \\
\hline \multirow[t]{3}{*}{ Media-A } & JM & & & $0.87(0.08)$ & & & $0.75(0.17)$ & & $0.78(0.11)$ & $0.86(0.11)$ & $0.89(0.10)$ \\
\hline & HD & & & $1.02(0.58)$ & & & $1.98(1.42)$ & & $1.94(1.02)$ & $1.27(0.90)$ & $1.05(0.84)$ \\
\hline & PAD & & & $0.09(0.09)$ & & & $0.22(0.16)$ & & $0.15(0.13)$ & $0.12(0.16)$ & $0.10(0.11)$ \\
\hline \multirow[t]{3}{*}{ Media-B } & JM & $0.78(0.11)$ & & $0.91(0.04)$ & $0.74(0.16)$ & & $0.85(0.09)$ & & $0.78(0.12)$ & $0.92(0.04)$ & $0.95(0.03)$ \\
\hline & HD & $0.57(0.39)$ & & $0.31(0.12)$ & $0.76(0.47)$ & & $0.53(0.37)$ & & $0.63(0.31)$ & $0.24(0.11)$ & $0.15(0.10)$ \\
\hline & PAD & $0.18(0.12)$ & & $0.04(0.04)$ & $0.22(0.20)$ & & $0.10(0.13)$ & & $0.18(0.16)$ & $0.06(0.04)$ & $0.03(0.04)$ \\
\hline
\end{tabular}




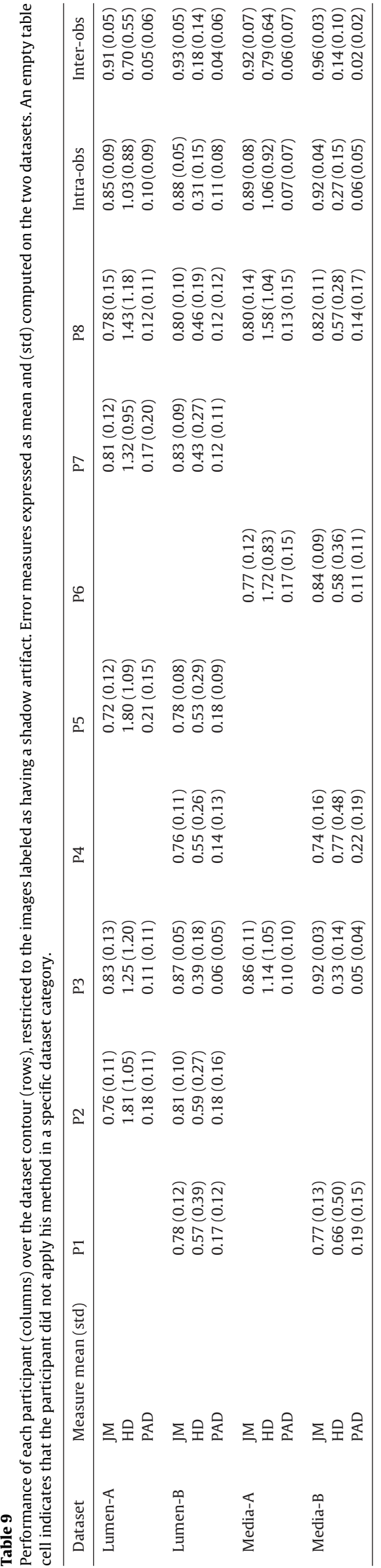

seven separated tables report the results of the algorithm considering only a distinct frame category: Plaque (Table 6), Bifurcations (Table 7), Stent (Table 10), Side vessels (Table 8), Shadow artifact (Table 9), Guide-wire artifact (Table 11), Catheter close to lumen (Table 12).

As detailed in Section 2.1.5, no frame has been assigned to three categories (stent, guide wire artifacts, catheter touching the lumen) for dataset $\mathrm{B}$, hence the corresponding lines are left empty.

The algorithm performance can be visually summarized by boxplots, which illustrate the median, first and third quartile and outliers for each method on the same figure. The box-plot in Figs. 3-5 reports the evaluation measure summarized in Table 5. Each figure is composed of four sub-plots corresponding to each dataset and to each segmentation class (lumen and media). An empty column indicates that the participant did not apply his method in a specific dataset category.

The analysis provided in Tables $5-12$ is complemented by statistical significance tests at $5 \%$ using a paired $T$-test, showing if the scoring difference between two algorithms is significantly different or not. In Table 13, for each combination of algorithms, the presence of the letter $\mathrm{J}, \mathrm{H}$ and $\mathrm{P}$ refers to statistical significance with respect to the Jaccard, Hausdorff Distance and PAD, respectively. The hyphen indicates that (at least) one of the two algorithms did not participate to the specific challenge, so that the comparison cannot be performed. The symbol $\star$ means that for none of the measures, the two algorithms present statistically significant different results. The three measures have been reported, because in some cases the significant difference is observed in only one or two of them. It can be concluded that the three performance measures are important and complementary when evaluating the algorithm performance, because in some case, a disagreement between the statistical significance of the three measures might be observed.

Comparing the entries of Table 5 with the entries of Tables $6-12$, it can be observed that the performance of the algorithms varies significantly depending on the type of image to segment. It is difficult to have a global overview only observing numerical results. A visual comparison is presented in Fig. 6. In this figure, the performance of each method, on the different image categories is reported on a normalized (between 0.7 and 1) circular plot. Hence, in Fig. 6 the Jaccard score is summarized for each category: General performance (S1), Plaque (S2), Bifurcations (S3), Side vessels (S4), Shadow artifact (S5), Stent (S6), Guide-wire artifact (S7), Catheter touching the lumen (S8). It can be observed that for instance, some algorithms (e.g. P1, P3, P6, P7 and P8) have similar performances on all the frames of the datasets, whereas other algorithms are more specific and their performance varies depending on the frame analyzed. It must be noted that the values are normalized, hence no direct comparison should be made among the columns of Table 6 . Observing Fig. 6, the IVUS images that turned out to be more difficult to segment are the bifurcations. The error might be caused by the ambiguous definition of the vessel in presence of a bifurcation. Some algorithms might choose to avoid the branching artery, some other to consider as vessel the area included in the branching vessel.

Finally, when it is required to analyze the performance of a method under a clinical prospective, it is interesting to know the number of frames, which fulfill a clinically relevant threshold. For instance, clinicians might consider the quality of a method according to the number of frames segmented with an error below a specific PAD threshold.

For this reason, Fig. 7 reports the percentage of frames, for each dataset, which lie below a progressive threshold of the PAD. Obviously, such measurement can be repeated for the other scores described in Section 2.1.6, however the results were similar and 
DATASET A LUMEN

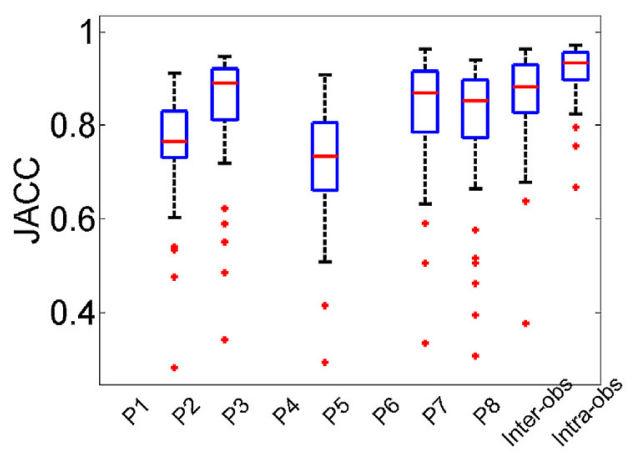

DATASET B LUMEN

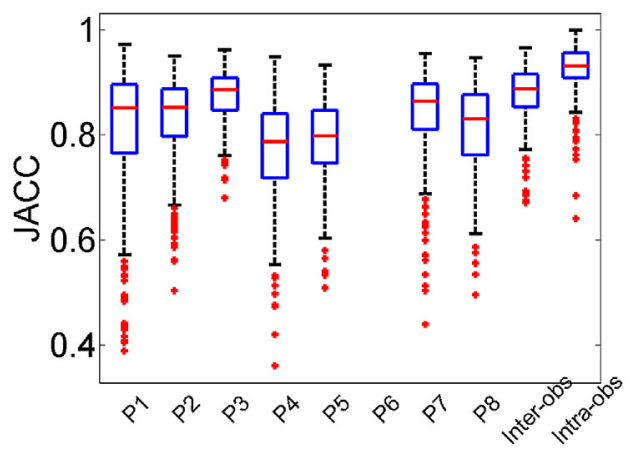

DATASET A MEDIA

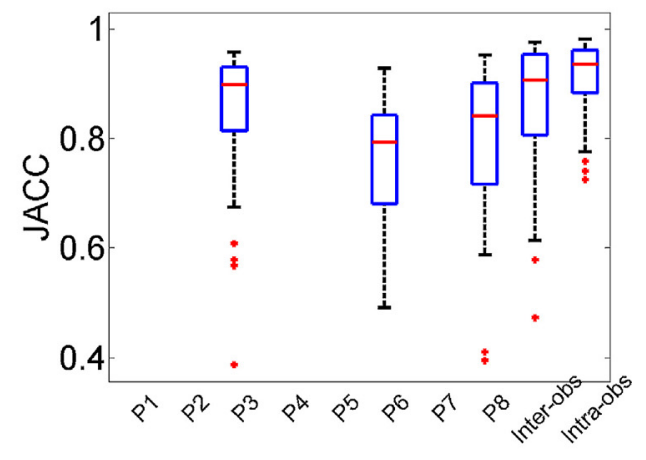

DATASET B MEDIA

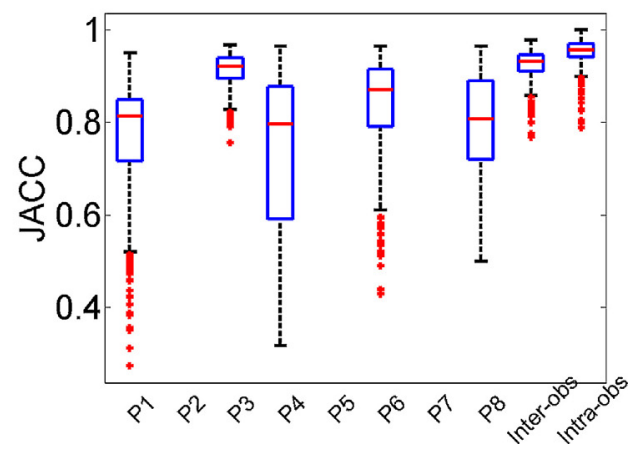

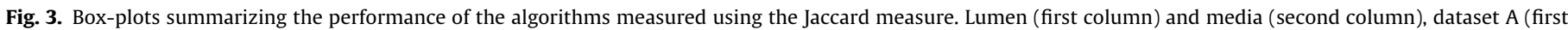
row) and dataset B (second row), respectively. An empty column indicates that the participant did not apply his method in a specific dataset category.

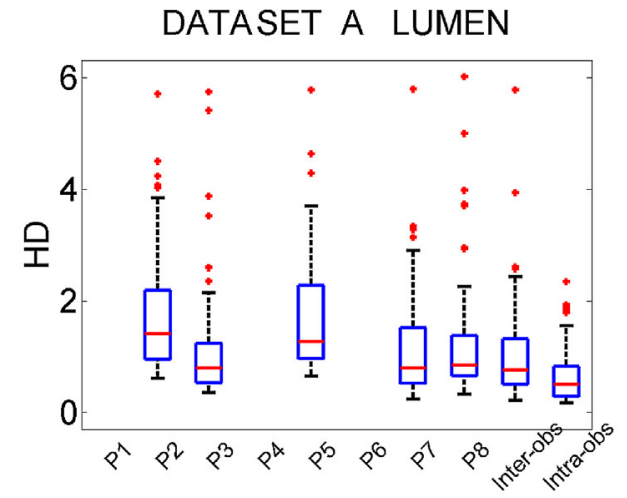

DATASET B LUMEN

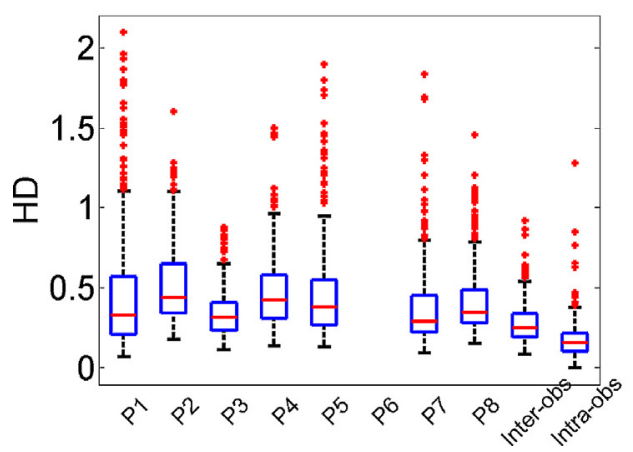

DATASET A MEDIA
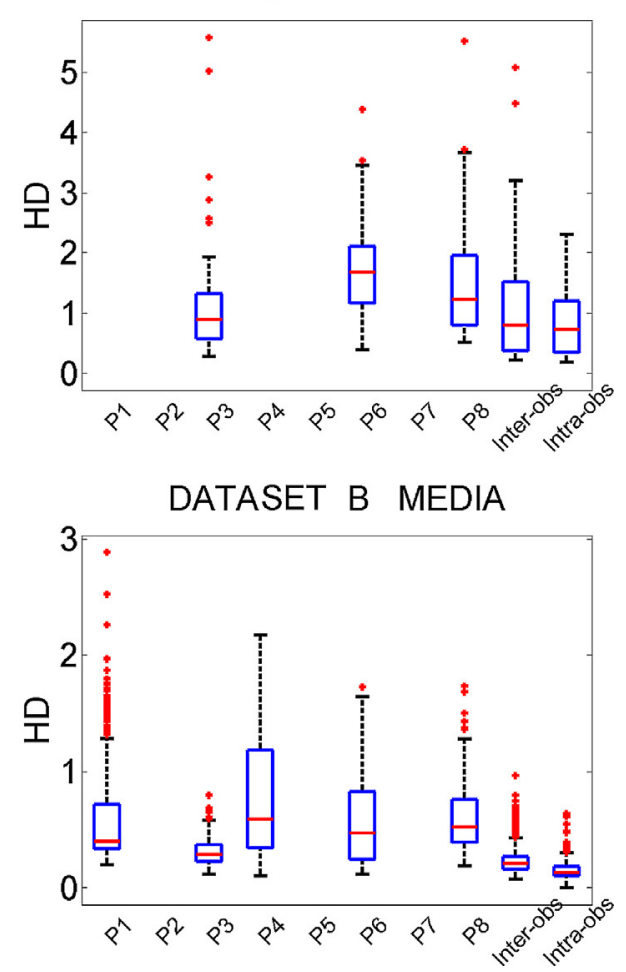

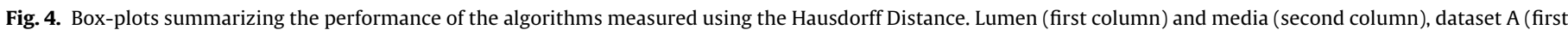
row) and dataset B (second row), respectively. An empty column indicates that the participant did not apply his method in a specific dataset category. 
DATASET A LUMEN

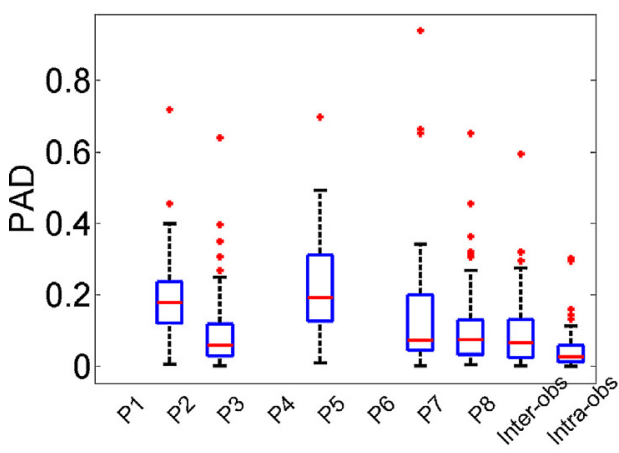

DATASET B LUMEN

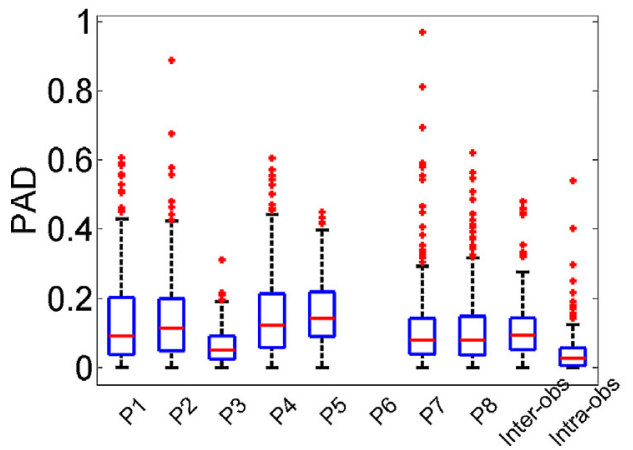

DATASET A MEDIA

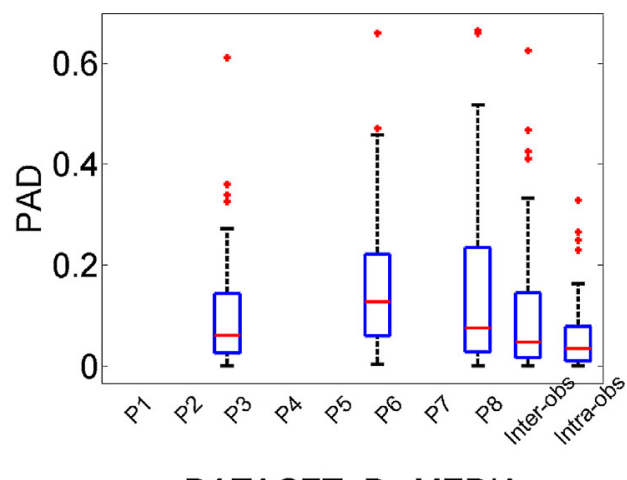

DATASET B MEDIA

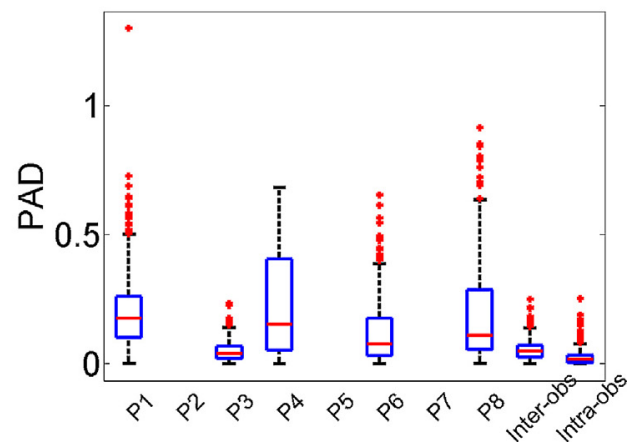

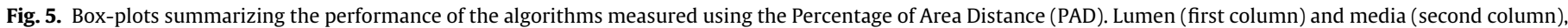
dataset A (first row) and dataset B (second row), respectively. An empty column indicates that the participant did not apply his method in a specific dataset category.

Table 10

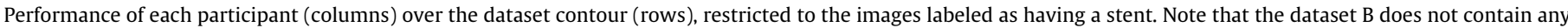

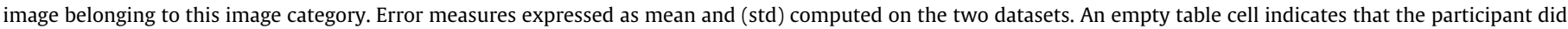
not apply his method in a specific dataset category.

\begin{tabular}{|c|c|c|c|c|c|c|c|c|c|c|c|}
\hline Dataset & Measure mean (std) & P1 & $\mathrm{P} 2$ & P3 & P4 & P5 & P6 & P7 & P8 & Intra-obs & Inter-obs \\
\hline \multirow[t]{3}{*}{ Lumen-A } & JM & & $0.73(0.09)$ & $0.83(0.09)$ & & $0.73(0.10)$ & & $0.83(0.12)$ & $0.82(0.11)$ & $0.86(0.09)$ & $0.91(0.08)$ \\
\hline & HD & & $2.00(1.28)$ & $1.36(0.95)$ & & $1.78(1.11)$ & & $1.12(0.95)$ & $1.37(1.22)$ & $1.02(0.75)$ & $0.71(0.65)$ \\
\hline & PAD & & $0.22(0.10)$ & $0.09(0.08)$ & & $0.21(0.13)$ & & $0.14(0.18)$ & $0.08(0.10)$ & $0.07(0.08)$ & $0.05(0.08)$ \\
\hline \multirow[t]{3}{*}{ Lumen-B } & JM & & & & & & & & & & \\
\hline & HD & & & & & & & & & & \\
\hline & PAD & & & & & & & & & & \\
\hline \multirow[t]{3}{*}{ Media-A } & JM & & & $0.87(0.08)$ & & & $0.75(0.12)$ & & $0.84(0.08)$ & $0.86(0.11)$ & $0.91(0.06)$ \\
\hline & HD & & & $1.03(0.58)$ & & & $1.81(0.89)$ & & $1.36(0.86)$ & $1.06(0.75)$ & $0.79(0.57)$ \\
\hline & PAD & & & $0.07(0.07)$ & & & $0.22(0.20)$ & & $0.08(0.08)$ & $0.13(0.15)$ & $0.07(0.07)$ \\
\hline \multirow[t]{3}{*}{ Media-B } & JM & & & & & & & & & & \\
\hline & HD & & & & & & & & & & \\
\hline & PAD & & & & & & & & & & \\
\hline
\end{tabular}

Table 11

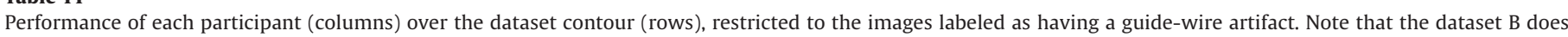

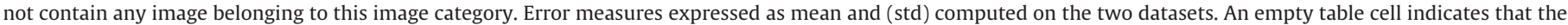
participant did not apply his method in a specific dataset category.

\begin{tabular}{|c|c|c|c|c|c|c|c|c|c|c|c|}
\hline Dataset & Measure mean (std) & P1 & $\mathrm{P} 2$ & P3 & P4 & P5 & P6 & P7 & P8 & Intra-obs & inter-obs \\
\hline \multirow[t]{3}{*}{ Lumen-A } & JM & & $0.76(0.09)$ & $0.87(0.11)$ & & $0.74(0.11)$ & & $0.86(0.11)$ & $0.81(0.14)$ & $0.88(0.08)$ & $0.93(0.05)$ \\
\hline & HD & & $1.40(0.80)$ & $1.02(1.15)$ & & $1.39(0.93)$ & & $0.98(0.90)$ & $1.16(1.12)$ & $0.92(0.81)$ & $0.56(0.44)$ \\
\hline & PAD & & $0.20(0.08)$ & $0.07(0.08)$ & & $0.21(0.11)$ & & $0.12(0.19)$ & $0.10(0.12)$ & $0.08(0.09)$ & $0.04(0.06)$ \\
\hline \multirow[t]{3}{*}{ Lumen-B } & JM & & & & & & & & & & \\
\hline & HD & & & & & & & & & & \\
\hline & PAD & & & & & & & & & & \\
\hline \multirow[t]{3}{*}{ Media-A } & JM & & & $0.84(0.11)$ & & & $0.77(0.10)$ & & $0.79(0.14)$ & $0.87(0.10)$ & $0.91(0.06)$ \\
\hline & HD & & & $1.24(1.02)$ & & & $1.55(0.55)$ & & $1.51(0.93)$ & $1.08(0.96)$ & $0.78(0.57)$ \\
\hline & PAD & & & $0.11(0.10)$ & & & $0.16(0.13)$ & & $0.15(0.19)$ & $0.13(0.16)$ & $0.07(0.08)$ \\
\hline \multirow[t]{3}{*}{ Media-B } & JM & & & & & & & & & & \\
\hline & HD & & & & & & & & & & \\
\hline & PAD & & & & & & & & & & \\
\hline
\end{tabular}


Table 12

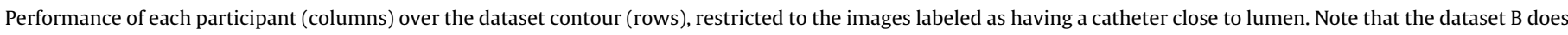

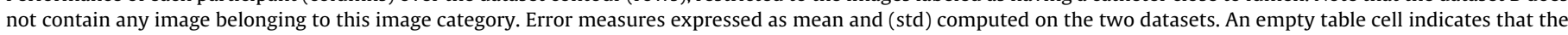
participant did not apply his method in a specific dataset category.

\begin{tabular}{|c|c|c|c|c|c|c|c|c|c|c|c|}
\hline Dataset & Measure mean (std) & $\mathrm{P} 1$ & $\mathrm{P} 2$ & P3 & P4 & P5 & P6 & P7 & P8 & Intra-obs & Inter-obs \\
\hline \multirow[t]{3}{*}{ Lumen-A } & JM & & $0.74(0.12)$ & $0.86(0.11)$ & & $0.73(0.12)$ & & $0.83(0.12)$ & $0.83(0.12)$ & $0.86(0.11)$ & $0.92(0.06)$ \\
\hline & HD & & $1.88(1.17)$ & $1.06(0.99)$ & & $1.63(1.07)$ & & $1.14(1.06)$ & $1.10(1.05)$ & $0.99(1.01)$ & $0.59(0.44)$ \\
\hline & PAD & & $0.21(0.14)$ & $0.10(0.12)$ & & $0.22(0.15)$ & & $0.13(0.14)$ & $0.11(0.14)$ & $0.10(0.12)$ & $0.04(0.06)$ \\
\hline \multirow[t]{3}{*}{ Lumen-B } & JM & & & & & & & & & & \\
\hline & HD & & & & & & & & & & \\
\hline & PAD & & & & & & & & & & \\
\hline \multirow[t]{3}{*}{ Media-A } & JM & & & $0.86(0.12)$ & & & $0.77(0.10)$ & & $0.81(0.13)$ & $0.86(0.13)$ & $0.92(0.07)$ \\
\hline & HD & & & $1.14(1.01)$ & & & $1.81(0.79)$ & & $1.48(0.96)$ & $1.17(1.05)$ & $0.80(0.56)$ \\
\hline & PAD & & & $0.10(0.13)$ & & & $0.16(0.15)$ & & $0.14(0.18)$ & $0.12(0.17)$ & $0.06(0.08)$ \\
\hline \multirow[t]{3}{*}{ Media-B } & JM & & & & & & & & & & \\
\hline & HD & & & & & & & & & & \\
\hline & PAD & & & & & & & & & & \\
\hline
\end{tabular}

thus they have been omitted due to page restrictions. It is interesting to note that for different PAD values some curves might cross. This means that for instance the method has lower performance in case of difficult frames (the ones having a low PAD), while improves with respect to the other methods when simple frames are segmented. The inter- and intra-observer variations were also determined, in order to have a general overview with respect to the manual annotation performances. In the case of the lumen, several

Table 13

Statistical significance results for: dataset A, lumen (a), dataset B, lumen (b), dataset A, media(c), dataset B, media (d), respectively.

\begin{tabular}{|c|c|c|c|c|c|c|c|c|c|c|}
\hline & P1 & P2 & P3 & P4 & P5 & P6 & P7 & P8 & Inter & Intra \\
\hline \multicolumn{11}{|l|}{ (a) } \\
\hline P1 & & JHP & JHP & - & JHP & - & JHP & JHP & JHP & JHP \\
\hline P2 & & & JHP & - & $\star$ & - & $\mathrm{JH}$ & JHP & JHP & JHP \\
\hline P3 & & & & - & JHP & - & JHP & JHP & $\mathrm{H}$ & JHP \\
\hline P4 & & & & & - & - & - & - & - & - \\
\hline P5 & & & & & & - & JHP & JHP & JHP & JHP \\
\hline P6 & & & & & & JHP & - & - & - & - \\
\hline P7 & & & & & & & & $\mathrm{P}$ & JHP & JHP \\
\hline P8 & & & & & & & & & JHP & JHP \\
\hline Inter & & & & & & & & & & JHP \\
\hline \multicolumn{11}{|l|}{ Intra } \\
\hline \multicolumn{11}{|l|}{ (b) } \\
\hline P1 & & JP & JHP & $\mathrm{J}$ & $\mathrm{JP}$ & - & JHP & HP & JHP & JHP \\
\hline P2 & & & $\mathrm{JH}$ & $\mathrm{JP}$ & JHP & - & JHP & $\mathrm{J}$ & $\mathrm{JH}$ & JHP \\
\hline P3 & & & & JHP & JHP & - & $\mathrm{JP}$ & JHP & $\mathrm{JH}$ & JHP \\
\hline P4 & & & & & HP & - & JHP & $\mathrm{JP}$ & JHP & JHP \\
\hline P5 & & & & & & - & JHP & JHP & JHP & JHP \\
\hline P6 & & & & & & & - & - & - & - \\
\hline P7 & & & & & & & & JHP & JHP & JHP \\
\hline P8 & & & & & & & & & $\mathrm{JH}$ & JHP \\
\hline Inter & & & & & & & & & & JHP \\
\hline \multicolumn{11}{|l|}{ Intra } \\
\hline \multicolumn{11}{|l|}{ (c) } \\
\hline P1 & & - & JHP & - & - & JHP & - & JHP & JHP & JHP \\
\hline P2 & & & - & - & - & - & - & - & - & - \\
\hline P3 & & & & - & - & JHP & - & $\mathrm{JH}$ & $\mathrm{JH}$ & JHP \\
\hline P4 & & & & & - & - & - & - & - & - \\
\hline P5 & & & & & & - & - & - & - & - \\
\hline P6 & & & & & & & - & $\star$ & JHP & JHP \\
\hline P7 & & & & & & & & - & - & - \\
\hline P8 & & & & & & & & & JHP & JHP \\
\hline Inter & & & & & & & & & & JHP \\
\hline \multicolumn{11}{|l|}{ Intra } \\
\hline \multicolumn{11}{|l|}{ (d) } \\
\hline P1 & & - & JHP & $\mathrm{H}$ & - & JHP & - & JHP & JHP & JHP \\
\hline P2 & & & - & - & - & - & - & - & - & - \\
\hline P3 & & & & JHP & - & JHP & - & JHP & $\mathrm{JH}$ & JHP \\
\hline P4 & & & & & - & JHP & - & JHP & JHP & JHP \\
\hline P5 & & & & & & - & - & - & - & - \\
\hline P6 & & & & & & & - & JP & JHP & JHP \\
\hline P7 & & & & & & & & - & - & - \\
\hline P8 & & & & & & & & & JHP & JHP \\
\hline Inter & & & & & & & & & & JHP \\
\hline Intra & & & & & & & & & & \\
\hline
\end{tabular}




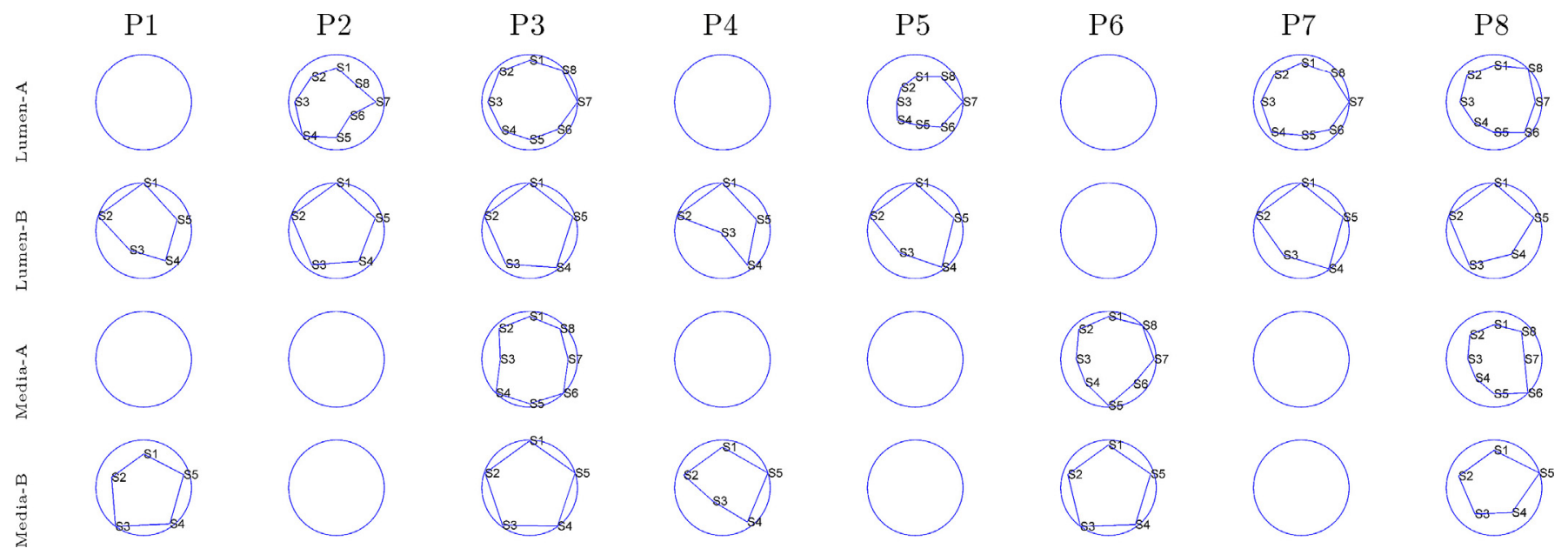

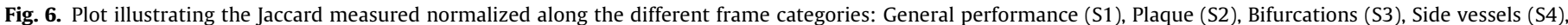

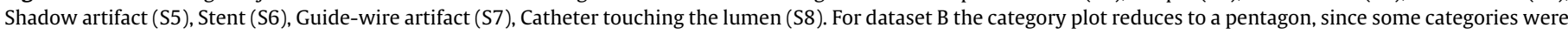
not present in the dataset (see Section 2.1.5). An empty plot indicates that the participant did not apply his method in a specific dataset category.

methods are as good as the inter-observer variability, while in the case of the media the segmentation task is more challenging.

\subsection{Qualitative evaluation}

A qualitative evaluation allows to complete the comparison among the algorithms, and to observe in details the performance of each method. For each dataset (Figs. 8 and 9 for dataset $A$ and $B$, respectively), six exemplar and challenging frames are chosen (columns), and the segmentation contours (in red and blue solid lines for media and lumen border, respectively) are superimposed with the ground truth annotations (in dotted yellow lines).
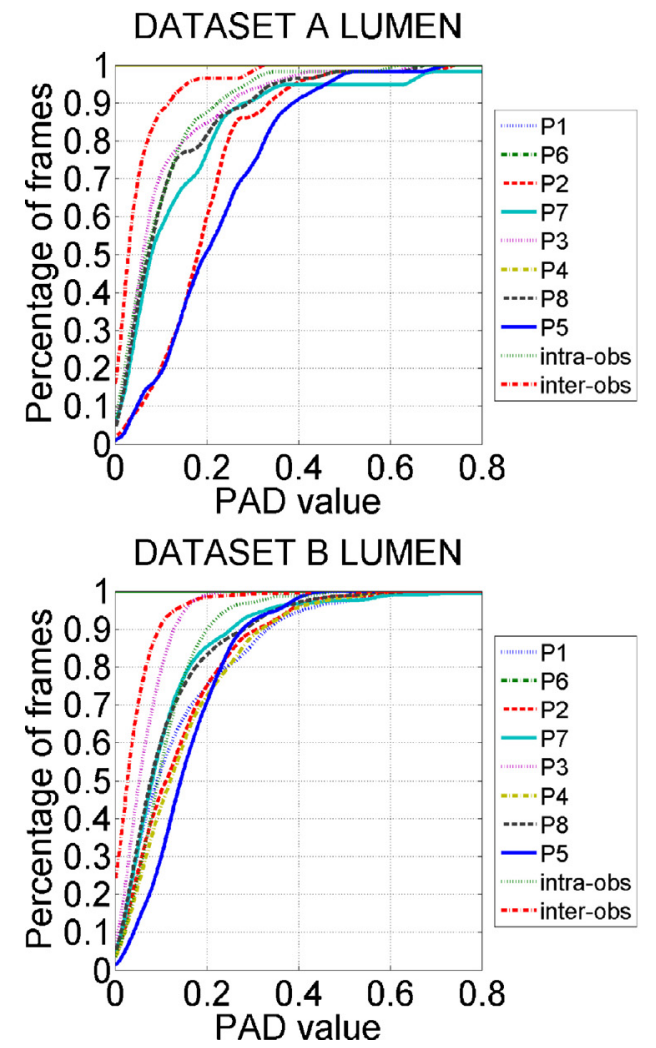

It is interesting to observe that, in general, for the lumen the most important errors were induced by the presence of the catheter shadow and stent (in dataset A), and by the similar echogenicity of the lumen and soft plaque texture (in both datasets). In the case of media, in most of the cases the calcium and catheter shadow are the major segmentation obstacles.

\section{Discussion}

The aim of the paper is to present a standardized evaluation methodology and reference database for evaluating IVUS image segmentation. Hence, in this manuscript, an extensive analysis of

\section{DATASET A MEDIA}

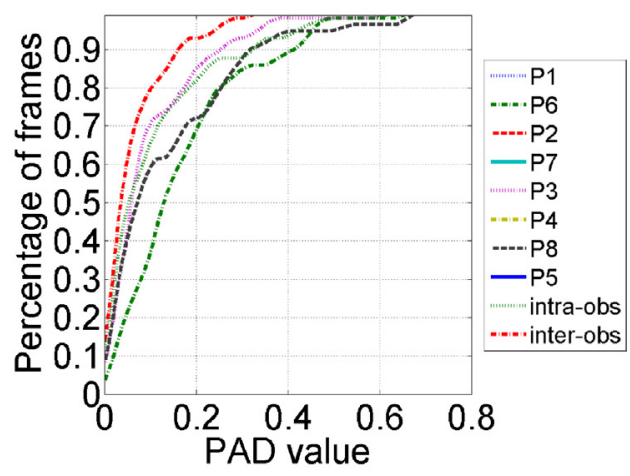

\section{DATASET B MEDIA}

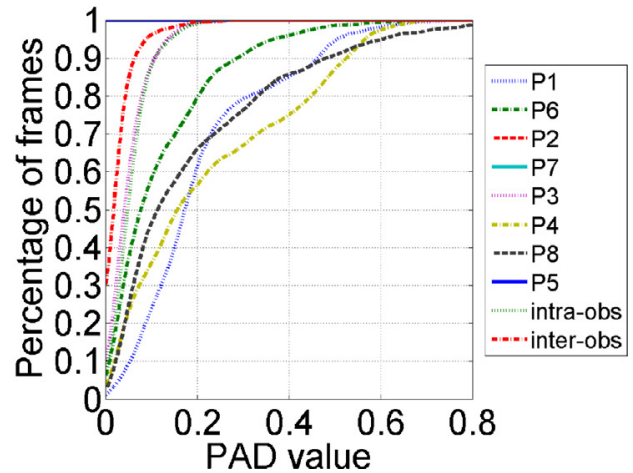

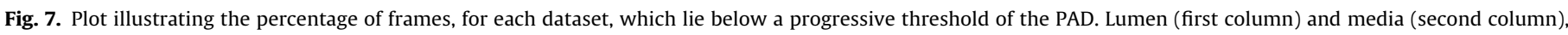
dataset A (first row) and dataset B (second row), respectively. 
P1

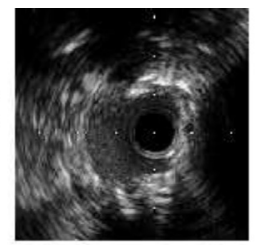

P2

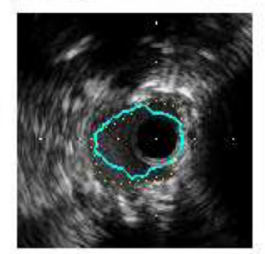

P3

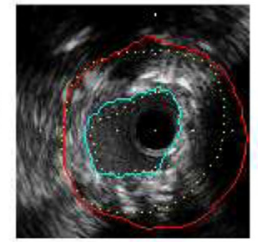

P4

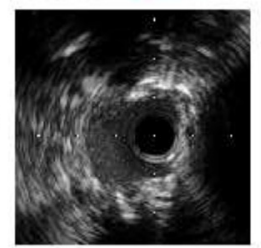

P5

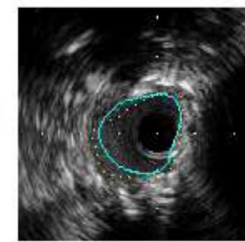

P6

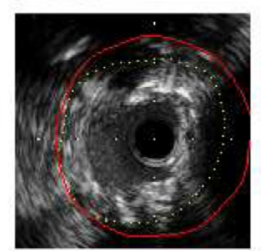

P7

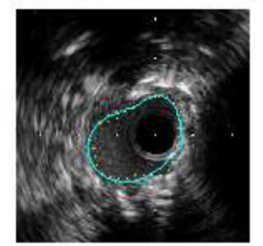

P8

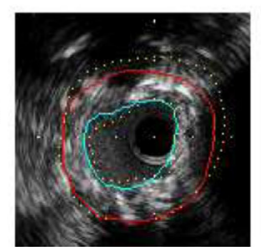

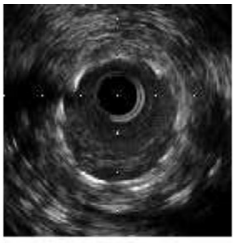
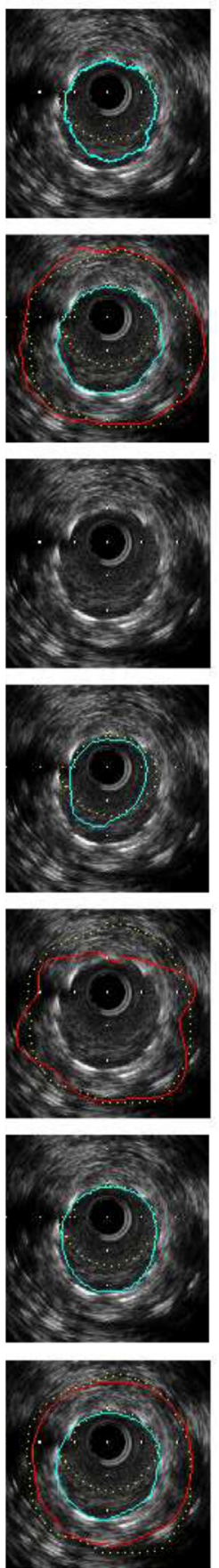
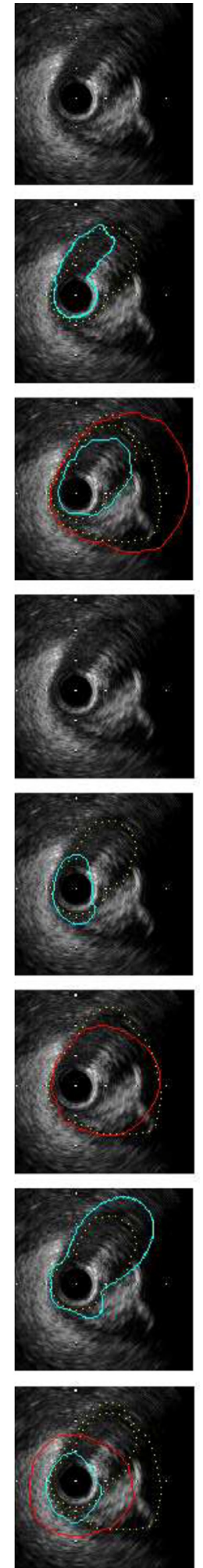
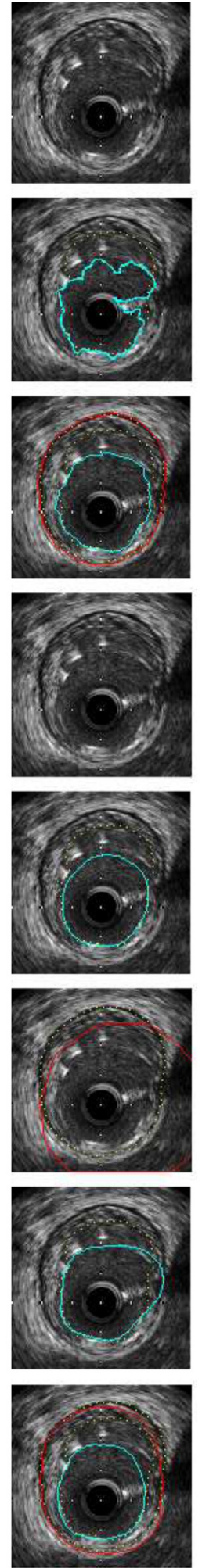
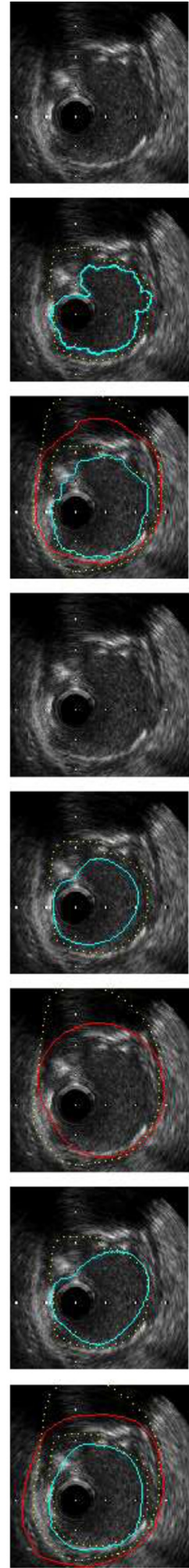
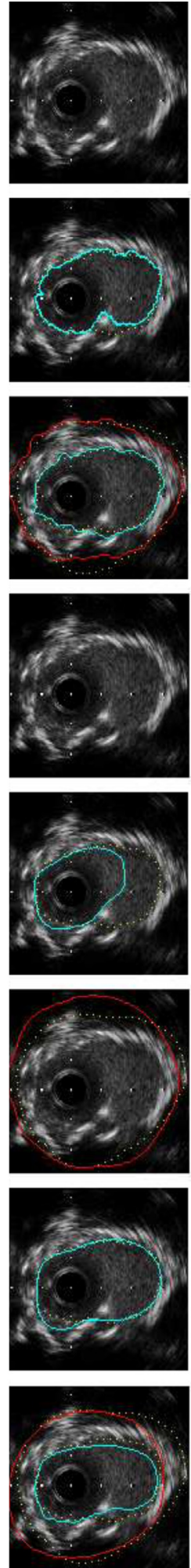

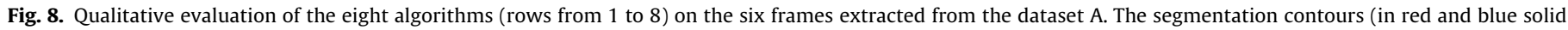

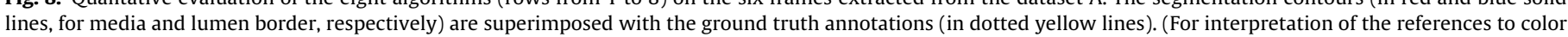
in this figure legend, the reader is referred to the web version of the article.)

the results by means of several tables and figures is performed. Although the detailed analysis of each method performance is out of the scope of this paper, some general conclusions can be drawn.

\subsection{Participant 1}

The semi-automatic method (in terms of intensity distribution initialization rather than a geometric contour input) has been 
P1

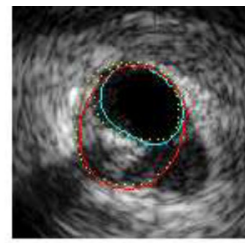

$\mathrm{P} 2$

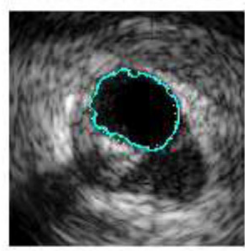

P3

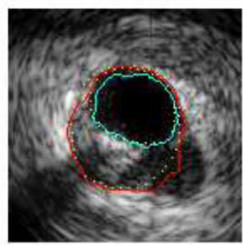

P4

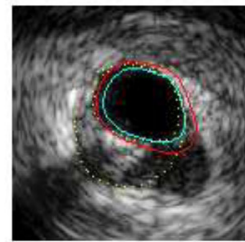

P5

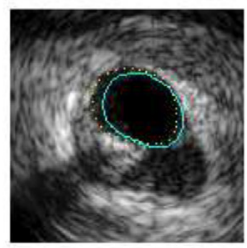

P6

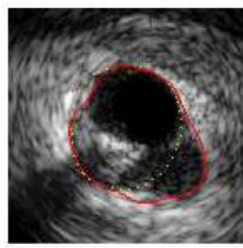

P7

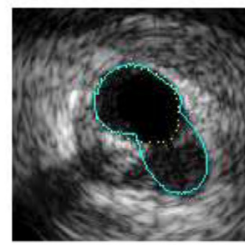

P8

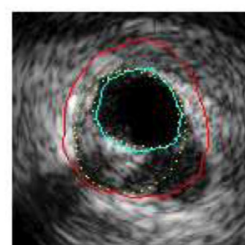

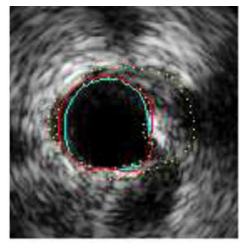
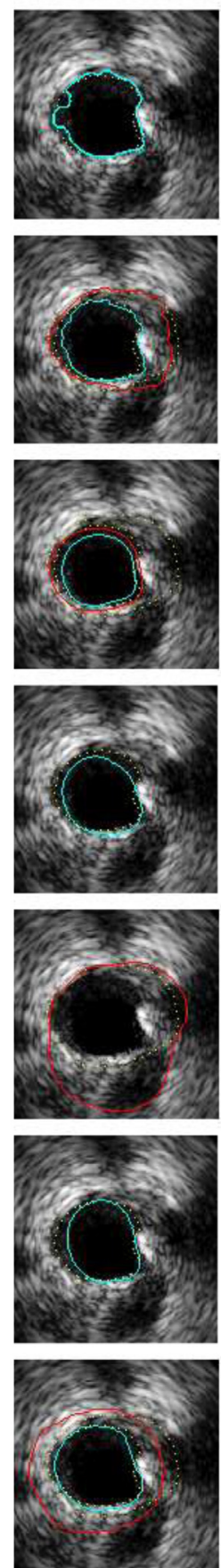
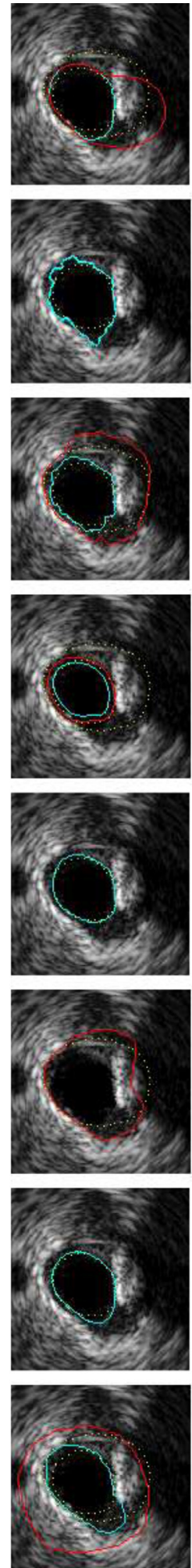
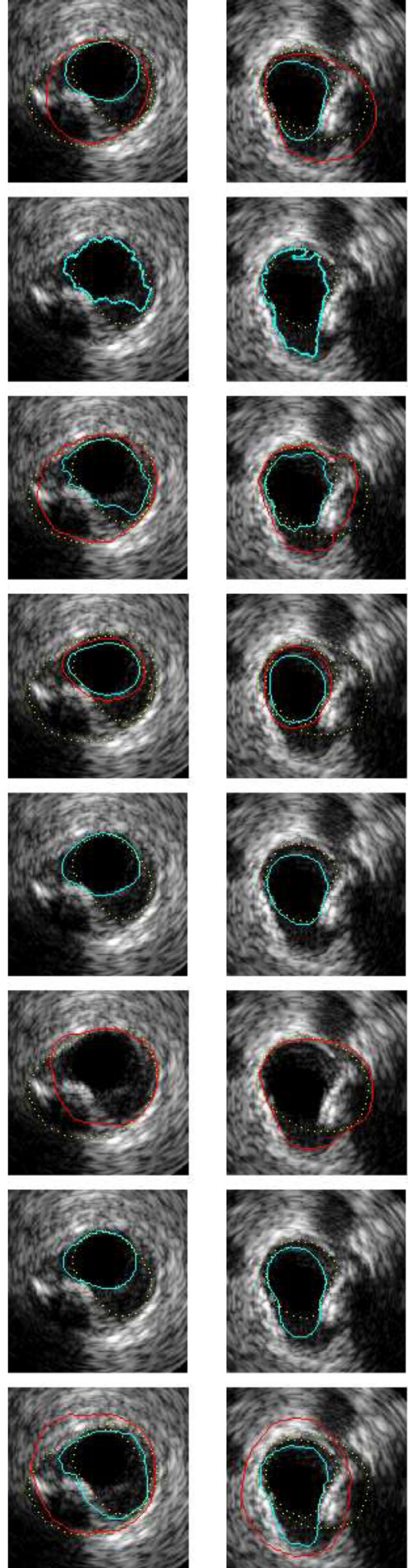
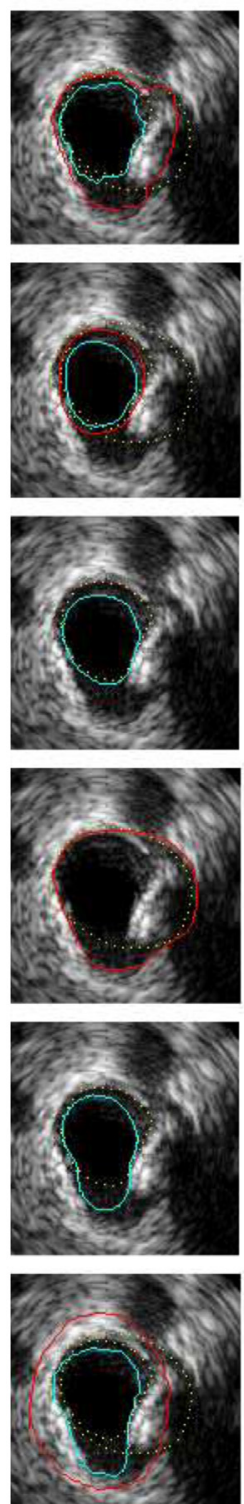
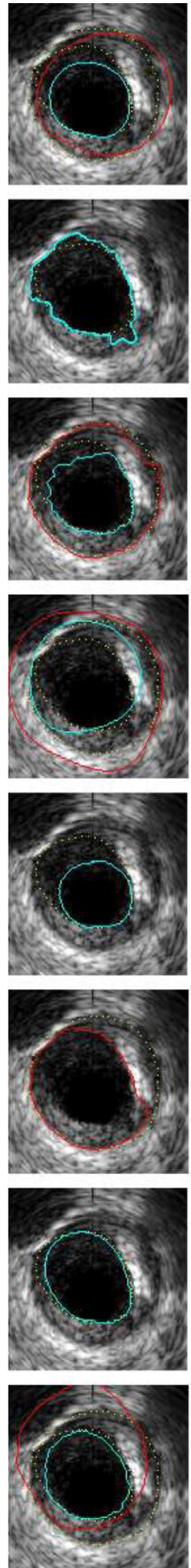

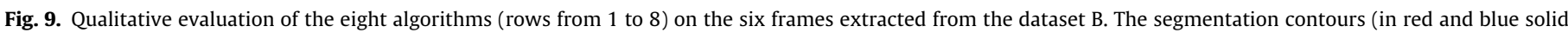

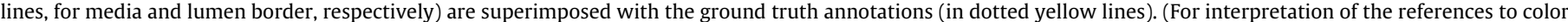
in this figure legend, the reader is referred to the web version of the article.)

specifically developed for the dataset $\mathrm{B}$, and provided reasonable results both for lumen an media contours (Figs. 3-5). The method exhibited slightly lower performance in the case of bifurcations, while the performance on the other frames is balanced (Fig. 6). In case of the media, the performance is lower for the most difficult frames while it improves over the easier frames (Fig. 7). The initialization of the algorithm requires less interactions with respect to those of $\mathrm{P} 2$. 


\subsection{Participant 2}

The semi-automatic method is able to provide the lumen contours both on datasets A and B (Figs. 3-5). The method performance is slightly lower in the presence of a stent, while the performance on the other frames is balanced (Fig. 6). In case of the dataset A, the performance is lower for the most difficult frames. However, the gap with the other methods is reduced in the case of easy frames (Fig. 7). The segmentation contours are wrinkled (Figs. 8 and 9), and a regularization might improve the final segmentation results.

\subsection{Participant 3}

The semi-automatic method provides the best performance for lumen and media contours both on dataset A and B (Figs. 3-5). The method performance is balanced on all the frames categories (Fig. 6). In case of the lumen and dataset B, the algorithm performance is better than the inter-observer variability and approaches the intra-observer results (Fig. 7). Such extremely good results might be a consequence of the semi-automatic initialization, which may play an important role in approximating the solution to the expected boundary, especially in the case of frames containing artifacts.

\subsection{Participant 4}

The fully-automatic method, has been applied only to the frames belonging to the dataset $\mathrm{B}$, and provided acceptable results both for lumen an media contours (Figs. 3-5). The method provides the lowest performances in case of bifurcations, (Fig. 6). However, the method was not designed to handle bifurcations (since bifurcation segmentation is treated separately in the semi-automated method described in [65]. In case of the media, the performance is lower with respect to the other methods for the easiest frames (Fig. 7).

\subsection{Participant 5}

The fully-automatic method, provides acceptable segmentation of the lumen contours in frames belonging to both datasets $A$ and B (Figs. 3-5). The method is particularly suited for frames having guide-wire artifacts, while the performance on other frames is balanced. The performance is lower with respect to the other methods for the easiest frames, and becomes comparable to the others for the most difficult frames (Fig. 7). However, in dataset A, the method performance is comparable with P2 (see the $\star$ in Table 13a) which is a semi-automatic method.

\subsection{Participant 6}

The fully-automatic method is devoted to media contours segmentation and has been applied to both datasets A and B (Figs. 3-5). Considering only the fully-automatic methods, this approach provides the best performance on the dataset $B$, and comparable results with respect to P8 for the datasets $\mathrm{A}$ (see the $\star$ in the Table 13c). Considering the overall performance, the method is second only to the P3, which proposed a semi-automatic method. The method scores are balanced on all the frames categories.

\subsection{Participant 7}

The semi-automatic method provides accurate lumen contours in frames belonging to both datasets A and B (Figs. 3-5). The method performance is balanced on all the frames categories. The performance tends to the inter-observer variability in dataset $\mathrm{B}$. The method reaches the second overall ranking in the lumen segmentation category for dataset B, and the third for the dataset A.
The initialization of the algorithm requires less interactions with respect to the $\mathrm{P} 2$.

\subsection{Participant 8}

The fully-automatic method, obtains very good lumen and media contours in frames belonging to both datasets $A$ and $B$ (Figs. 3-5). The method performance is particularly good on shadow artifact and stent categories, while the score is lower when the method is applied to other images. The performance almost reaches the inter-observer variability in the case of lumen segmentation, while in the case of media the algorithm score is comparable (in dataset A) or lower (in dataset B) than P6.

\section{Conclusion}

This paper describes a novel evaluation framework that allows a standardized and objective quantitative comparison of IVUS lumen and media segmentation algorithms. The data collection is aimed at creating a reference standard and the evaluation benchmark for future segmentation techniques. The two datasets are composed of IVUS images acquired using different imaging and at different central frequencies, resulting in a heterogeneous data collection. Each frame is individually labeled according to separate image categories in order to assess robustness of an algorithm to morphological and imaging artifacts. Each dataset has been segmented by expert physicians, providing inter- and intra-variability reference annotations. Three evaluation measures have been established to quantitatively assess the methods performance, under a clinical and technological prospective.

This framework has been introduced at the MICCAI 2011 Computing and Visualization for (Intra)Vascular Imaging workshop, and the results of the eight algorithms, (either semi- or fullyautomatic) have been computed and compared. As illustrated in the manuscript, the framework allows the evaluation of an IVUS segmentation method, and extensive performance comparison between algorithms.

All algorithms presented to the IVUS segmentation challenge have advantages and drawbacks, as detailed in the discussion. For instance, a semi-automatic algorithm might allow reaching better performances but requires manual interaction. A 3D algorithm might be more robust because it exploits both spatial and temporal information, however it might require more memory for the processing. A supervised approach based on classification might require less tuning and can be easily generalized to different dataset, but the creation of a training-set is sometimes time-consuming. The methodologies proposed by the participants were heterogeneous; hence it would be difficult to combine all the advantages in a single approach. In order to be fair with the participant, no subjective judgment is made by the authors, and the final evaluation is left to the reader.

The framework allows an easy extension of new datasets, provided that sufficiently large number of image samples from different pullback sections with different characteristics, along with manual annotations of both lumen and media contours are available. In order to allow comparison of future segmentation algorithm a website has been created [56].

\section{Acknowledgments}

T. P. E. and D. I. F. are partially funded by ARTREAT, FP7-224297. G.C. and F.D. are partially funded by MDEIE, Canada; Boston Scientific, Fremont, CA, USA; NSERC (grant \#138570). B.S., F.C. and M.A. are partially funded by TIN2009-14404-C02; TIN2012-38187C03-01; Boston Scientific, USA and SGR00696. C. G. is supported 
by MICINN (Ramon y Cajal Grant). The work of C. W. W. and H. C. C. is partially funded by NSC, 101-2628-E-011-006-MY3. A.W. and R.W.D.: National Institutes of Health, U.S.A. (R01EB004640, R01HL063373). T.K.: Czech Ministry of Health, Czech Republic (IGA NR9214-3). E. G. M. was supported by CONACYT. I. A. K. was partially supported by NSF Grant DMS-0915242 and the UH Hugh Roy and Lillie Cranz Cullen Endowment Fund.

Any opinion, finding, conclusion or recommendation expressed in this material is of the authors and may not reflect the views of the sponsors.

\section{References}

[1] Morabia AAT. The who report preventing chronic diseases: a vital investment and us. Soz Praventivmed 2005;51(2):74.

[2] Frostegard J. Sle, atherosclerosis and cardiovascular disease. J Intern Med 2005;257(6):1365-2796.

[3] Glagov S, Weisenberg E, Zarins C, Stankunavicius R, Kolettis G. Compensatory enlargement of human atherosclerotic coronary arteries. N Engl J Med 1987;316(22):1371-5

[4] Wahle A, Wellnhofer E, Mugaragu I, Saner HU, Oswald H, Fleck E. Assessment of diffuse coronary artery disease by quantitative analysis of coronary morphology based upon 3-d reconstruction from biplane angiograms. IEEE Trans Med Imaging 1995;14(2):230-641.

[5] Nair A, Kuban BD, Tuzcu EM, Schoenhagen P, Nissen SE, Vince DG. Coronary plaque classification with intravascular ultrasound radiofrequency data analysis. Circulation 2002;106:2200-6.

[6] Sathyaranayana S, Carlier S, Wenguang L, Thomas L. Characterization of atherosclerotic plaque by spectral similarity of radiofrequency intravascular ultrasound signals. Eurointervention 2009;5:133-9.

[7] Wahle A, Lopez JJ, Olszewski ME, Vigmostad SC, Chandran KB, Rossen JD, et al. Plaque development, vessel curvature, and wall shear stress in coronary arteries assessed by X-ray angiography and intravascular ultrasound. Med Image Anal 2006;10(4):615-31.

[8] Balocco S, Gatta C, Alberti M, Carrillo X, Rigla J, Radeva P. Relation between plaque type, plaque thickness, blood shear stress, and plaque stress in coronary arteries assessed by X-ray angiography and intravascular ultrasound. Med Phys 2012;39(12):7430-45

[9] Katouzian A, Angelini ED, Carlier SG, Suri JS, Navab N, Laine AF. A state-ofthe-art review on segmentation algorithms in intravascular ultrasound (IVUS) images. IEEE Trans Inf Technol Biomed 2012;16(5):823-34

[10] Zhang X, McKay C, Sonka M. Tissue characterization in intravascular ultrasound images. IEEE Trans Med Imaging 1998;17(December):889-99.

[11] Sonka M, Zhang X, Siebes M, Bissing M, DeJong S, Collins S. Segmentation of intravascular ultrasound images: a knowledge-based approach. IEEE Trans Med Imaging 1995;14(December):719-32.

[12] Klingensmith JD, Shekhar R, Vince DG. Evaluation of three-dimensional segmentation algorithms for the identification of luminal and medialadventitial borders in intravascular ultrasound images. IEEE Trans Med Imaging 2000;19(October):996-1011.

[13] Kovalski G, Beyar R, Shofti R, Azhari H. Three-dimensional automatic quantitative analysis of intravascular ultrasound images. Ultrasound Med Biol 2000;26(4):527-37

[14] Plissiti M, Fotiadis D, Michalis L, Bozios G. An automated method for lumen and media-adventitia border detection in a sequence of IVUS frames. IEEE Trans Inf Technol Biomed 2004;8:131-41.

[15] Luo Z, Wang Y, Wang W. Estimating coronary artery lumen area with optimization-based contour detection. IEEE Trans Med Imaging 2003;22(April):546-64

[16] Hui-Zhu H, Liang Y, Friedman M. IVUS image segmentation based on contrast. In: Proc. SPIE medical imaging: image processing, vol. 4684. 2002. p. 1727-33.

[17] Brusseau E, de Korte C. Fully automatic luminal contour segmentation in intracoronary ultrasound imaging - a statistical approach. IEEE Trans Med Imaging 2004;23:554-66.

[18] Gil D, Hernandez A, Rodriguez O, Mauri J, Radeva P. Statistical strategy for anisotropic adventitia modelling in IVUS. IEEE Trans Med Imaging 2006;25(6):768-78

[19] Roy Cardinal M-H, Meunier J, Soulez G, Maurice R, Therasse E, Cloutier G. Intravascular ultrasound image segmentation: a three-dimensional fastmarching method based on gray level distributions. IEEE Trans Med Imaging 2006;25(5):590-601.

[20] dos Santos Filho E, Yoshizawa M, Tanaka A, Saijo Y. A study on intravascular ultrasound image processing. Rec Electr Commun Eng Conversazione, Tohoku Univ 2006;74(2):30-3

[21] Unal G, Bucher S, Carlier S, Slabaugh G, Fang T, Tanaka K. Shape-driven segmentation of the arterial wall in intravascular ultrasound images. IEEE Trans Inf Technol Biomed 2008;12:335-47.

[22] Taki A, Najafi Z, Roodaki A, Setarehdan SK, Zoroofi RA, Konig A, et al. Automatic segmentation of calcified plaques and vessel borders in IVUS images. Int J Comput Assist Radiol Surg 2008;3(June):347-54.

[23] Downe R, Wahle A, Kovarnik T, Skalicka H, Lopez J, Horak J, et al. Segmentation of intravascular ultrasound images using graph search and a novel cost function. In: Proc. 2nd MICCAI workshop on computer vision for intravascular and intracardiac imaging. 2008. p. 71-9.

[24] Papadogiorgaki M, Mezaris V, Chatzizisis YS, Giannoglou GD, Kompatsiaris I. Image analysis techniques for automated IVUS contour detection. Ultrasound Med Biol 2008;34(September):1482-98.

[25] Katouzian A, Baseri B, Konofagou E, Laine A. Automatic detection of blood versus non-blood regions on intravascular ultrasound (ivus) images using wavelet packet signatures. In: Proc. SPIE medical imaging 2008: ultrasonic imaging and signal processing. 2008.

[26] Mendizabal-Ruiz E, Rivera M, Kakadiaris I. Segmentation of the luminal border in intravascular ultrasound b-mode images using a probabilistic approach. Med Image Anal 2013;17(6):649-70.

[27] Mendizabal-Ruiz E, Kakadiaris I. Probabilistic segmentation of the lumen from intravascular ultrasound radio frequency data. In: International Conference on Medical Image Computing and Computer Assisted Intervention. 2012 [in press].

[28] Ciompi F, Pujol O, Fernandez-Nofrerias E, Mauri J, Radeva P. Ecoc random fields for lumen segmentation in radial artery ivus sequences. Med Image Comput Comput Assist Interv 2009;5762:869-76.

[29] Wennogle M, Hoff W. Three dimensional segmentation of intravascular ultrasound data. In: Proc. 6th international conference on image analysis and recognition. 2009. p. 772-81.

[30] Roy Cardinal M-H, Soulez G, Tardif J, Meunier J, Cloutier G. Fast-marching segmentation of three-dimensional intravascular ultrasound images: a pre-and post-intervention study. Med Phys 2010;37(7):3633-47.

[31] Moraes M, Furuie S. Automatic coronary wall segmentation in intravascular ultrasound images using binary morphological reconstruction. Ultrasound Med Biol 2011;37:1486-99.

[32] Zhua X, Zhangc P, Shaoa J, Chenga Y, Zhangc Y, Bai J. A snake-based method for segmentation of intravascular ultrasound images and its in vivo validation. Ultrasonics 2011;51(2):181-9.

[33] Sun Z, Liu C. A parallel method for segmenting intravascular ultrasound image sequence. Appl Mech Mater 2011;130:2051-5.

[34] Balocco S, Gatta C, Ciompi F, Pujol O, Carrillo X, Mauri J, et al. Combining growcut and temporal correlation for ivus lumen segmentation. In: Lecture Notes in Computer Science, 6669. 2011. p. 556-63.

[35] Vezhnevets V, Konouchine V. Grow-cut - interactive multi-label n-d image segmentation; 2005. p. 150-6.

[36] Sanz-Requena R, Moratal D, García-Sánchez DR, Bodí V, Rieta JJ, Sanchis JM. Automatic segmentation and 3d reconstruction of intravascular ultrasound images for a fast preliminary evaluation of vessel pathologies. Comput Med Imaging Graph 2007;31:71-80.

[37] Sun F, Liu Z, Li Y, Babyn P, Yao G, Zhang Y. Improved T-snake model based edge detection of the coronary arterial walls in intravascular ultrasound images. In: Bioinformatics and biomedical engineering, no. 1. 2009. p. 1-4.

[38] Zheng M, Yubin W, Yousheng W, Xiaodi S, Yali W. Detection of the lumen and media-adventitia borders in IVUS imaging. Int Conf Signal Process 2008;2(1):1059-62.

[39] Herrington DM, Johnson T, Santago P, Snyder WE. Semi-automated boundary detection for intravascular ultrasound. In: Comput Cardiol. 1992. p. 103-6.

[40] Moraes MC, Furuie SS. An automatic media-adventitia border segmentation approach for IVUS images. Comput Cardiol 2010:389-92.

[41] Zhu H, Liang Y, Friedman MH. IVUS image segmentation based on contrast. SPIE 2002:4684:1727-33.

[42] Giannoglou GD, Chatzizisis YS, Koutkias V, Kompatsiaris I, Papadogiorgaki M, Mezaris V, et al. A novel active contour model for fully automated segmentation of intravascular ultrasound images: in vivo validation in human coronary arteries. Comput Biol Med 2007;37(September):1292-302.

[43] Iskurt A, Candemir S, Akgul YS. Identification of luminal and medial adventitial borders in intravascular ultrasound images using level sets. Lecture Notes Comput Sci 2006:572-82.

[44] Mojsilovic A, Popovic M, Amodaj N, Babic R, Ostojic M. Automatic segmentation of intravascular ultrasound images: a texture-based approach. Ann Biomed Eng 1997;25(November):1059-71.

[45] Otsu N. A threshold selection method from gray-level histograms. IEEE Trans Syst Man Cybern 1979;9(1):62-6.

[46] Bovenkamp EGP, Dijkstra J, Bosh JG, Reiber JHC. Multi-agent segmentation of IVUS images. Pattern Recogn 2004;37(April):647-63.

[47] Olszewski M, Wahle A, Mitchell S, Sonka M. Segmentation of intravascular ultrasound images: a machine learning approach mimicking human vision. In: International congress series, 1268. 2004. p. 1045-9.

[48] Dijkstra J, Koning G, Tuinenburg J, Oemrawsingh P, Reiber J. Automatic border detection in intravascular ultrasound images for quantitative measurements of the vessel, lumen and stent parameters. Comput Cardiol 2001;1230:25-8.

[49] Schaap M, Metz CT, van Walsum T, van der Giessen AG, Weustink AC, Mollet NR, et al. Standardized evaluation methodology and reference database for evaluating coronary artery centerline extraction algorithms. Med Image Anal 2009;13(October):701-14.

[50] Hameeteman K, Zuluaga MA, Freiman M, Joskowicz L, Cuisenaire O, Valencia LF, et al. Evaluation framework for carotid bifurcation lumen segmentation and stenosis grading. Med Image Anal 2011;15(August):477-88.

[51] Heimann T, van Ginneken B, Styner MA, Arzhaeva Y, Aurich V, Bauer C, et al. Comparison and evaluation of methods for liver segmentation from ct datasets. IEEE Trans Med Imaging 2009;28(August):1251-65.

[52] Niemeijer M, van Ginneken B, Cree M, Mizutani A, Quellec G, Sanchez C, et al. Retinopathy online challenge: automatic detection of microaneurysms in digital color fundus photographs. IEEE Trans Med Imaging 2010;29(1):185-95. 
[53] van Ginneken B, Armato SG, de Hoop B, van Amelsvoort-van de Vorst S, Duindam T, Niemeijer $\mathrm{M}$, et al. Comparing and combining algorithms for computer-aided detection of pulmonary nodules in computed tomography scans: The anode09 study. Med Image Anal 2010;14(December):707-22.

[54] http://vpa.sabanciuniv.edu/sites/cvii2011/.

[55] http://cbl.uh.edu/challenges.

[56] https.//www.cvc.uab.es/IVUSchallenge2011/.

[57] Gatta C, Balocco S, Ciompi F, Hemetsberger R, Rodriguez-Leor O, Radeva P. Real-time gating of IVUS sequences based on motion blur analysis: method and quantitative validation. In: MICCAI 2010, LNCS 6362/2010. 2010. p. 59-67.

[58] Isguder G, Unal G, Groher M, Navab N, Kalkan A, Degertekin M, et al. Manifold learning for image-based gating of ivus pullback sequences. Springer Lecture Notes Comput Sci 2010;6326:139-48

[59] O’Malley SM, Granada JF, Carlier S, Naghavi M, Kakadiaris IA. Image-based gating of intravascular ultrasound pullback sequences. TITB 2008;12(3):299-306.

[60] Caselles V, Kimmel R, Sapiro G. Geodesic active contours. Int J Comput Vision 1997;22(1):61-79.

[61] Kang L, Xiaodong W, Chen DZ, Sonka M. Optimal surface segmentation in volumetric images-a graph-theoretic approach. IEEE Trans Pattern Anal Mach Intell 2006;28(1):119-34

[62] Gatta C, Puertas E, Pujol O. Multi-scale stacked sequential learning. Pattern Recogn 2011;44(10-11):2414-26

[63] Ciompi F, Pujol O, Gatta C, Alberti M, Balocco S, Carrillo X, et al. Holimab: a holistic approach for media-adventitia border detection in intravascular ultrasound. Med Image Anal 2012;16(6):1085-100.

[64] Bourantas CV, Kalatzis FG, Papafaklis MI, Fotiadis DI, Tweddel AC, Kourtis IC, et al. Angiocare: an automated system for fast three-dimensional coronary reconstruction by integrating angiographic and intracoronary ultrasound data. Catheter Cardiovasc Interv 2008;72(2):166-75.

[65] Downe RW, Wahle A, Garvin JW, Kovarnik T, Horak J, Lopez JJ, et al. Identification and 3-d modeling of coronary branches in intravascular ultrasound. In: MICCAI workshop in computing and visualization for (intra)vascular imaging (CVII). 2011. p. 15-22

Simone Balocco teaches at University de Barcelona and is researcher at the Computer Vision Center, Bellaterra. He was a posdoctoral fellow at the Pompeu Fabra University, Barcelona in the CISTIB group. He obtained a PhD degree in Acoustics at CREATIS , University Lyon1, Lyon (France) and Ph.D. in Electronic and Telecommunication in MSD Lab, University of Florence (Italy). Simone has obtained his master thesis at CREATIS Lyon and his electronics degrees at the Politecnico of Turin (Italy). His research interests are: vascular modelling, image processing and machine learning techniques applied to Ultrasound and Magnetic Resonance and image-based assessment of tissue properties.

Carlo Gatta obtained the degree in Electronic Engineering in 2001 from the UniversitA degli Studi di Brescia (Italy). In 2006 he received the Ph.D. in Computer Science at the UniversitA degli Studi di Milano (Italy), with a thesis on perceptually based color image processing. In September 2007 he joined the Computer Vision Center at Universitat Automona de Barcelona (UAB) as a postdoc researcher working mainly on medical imaging. He is member of the Computer Vision Center and the BCN Perceptual Computing Lab. He is currently a senior researcher at the Computer Vision Center, under the Ramon y Cajal program. His main research interests are image processing, medical imaging, computer vision, machine learning and contextual learning.

Francesco Ciompi received the MSC degree in Electronic Engineering from the University of Pisa in 2006 and the MSC in Computer Vision and Artificial Intelligence from the Autonomous University of Barcelona in 2008. He obtained the PhD (cum laude) in Applied Mathematics and Analysis at Universitat de Barcelona in 2012. Since 2007, he is also member of the Computer Vision Center. His research interests include techniques of machine learning and context-aware models applied to segmentation and classification in medical images.

Andreas Wahle received an M.Sc. degree in Computer Science (1991) and a Ph.D. degree in Engineering (1997) from the Technical University of Berlin, Germany. He has been with the University of Iowa Department of Electrical and Computer Engineering since. His research focuses on medical image analysis and geometrical modeling in the cardiac and cardiovascular domains as well as image-data management in ophthalmology. He is a Senior Member of IEEE and a Member of SPIE.

Dr. Petia Radeva (PhD 1998, Universitat Autònoma de Barcelona, Spain) is a senior researcher and associate professor at the University of Barcelona. She is the head of Barcelona Perceptual Computing Laboratory (BCNPCL) at the University of Barcelona and the head of MiLab of Computer Vision Center. Her present research interests are on development of learning-based approaches (in particular, statistical methods) for computer visión and medical imaging. She is currently leading the projects: Machine learning tools for large scale object recognition, Audience measurements, Intestinal Motility Analysis in Wireless Endosccopy, Automatic Stent Detection in IVUS, Polyp detection, etc.

Stéphane Carlier received his MD degree from the Université Libre de Bruxelles (Belgium) in 1996 and defended his PhD in Biomedical Engineering at Erasmus University, Rotterdam (Netherlands) in 2001. He is currently interventional cardiologist at the University Hospital (UZ) Brussel. He held previously the position of Assistant Professor of Clinical Medicine and Bioengineering at the Columbia University, New York (NY) and Director of the Intravascular Imaging \& Physiology Corelab of the
Cardiovascular Research Foundation. His research interests include new intravascular imaging, cardiac and vascular dynamics, physiology and signal processing. He serves on several reviewing boards of medical and bioengineering journals.

Gozde Unal received her PhD in ECE with a minor in mathematics from North Carolina State University, NC, USA, in 2002. After a postdoctoral fellowship position at Georgia Institute of Technology, GA, USA, she worked as a research scientist at Siemens Corporate Research, Princeton, NJ, USA, between 2003-2007. She is currently an associate professor at Sabanci University, Faculty of Engineering and Natural Sciences, Istanbul, Turkey. Her research work is focused on mathematical solutions to medical image computing problems in neuroimaging and cardiovascular imaging with modalities such as MRI, diffusion MRI, CT, IVUS and Xray

Elias A. Sanidas, MD, PhD, FESC, FACC, is an interventional cardiologist born in 1973 in Greece. He studied medicine in Athens, Greece and completed his post-graduation training in the USA and in Belgium. The main field of his scientific work is invasive assessment of coronary anatomy and physiology including quantitative coronary angiography, intravascular ultrasound, optical coherence tomography and fractional flow reserve.

Josepa Mauri, MD., Ph.D. is an invasive cardiologist. She graduated in 1982 at Universitat Autonoma of Barcelona, with PhD in 1992 at Universitat de Barcelona. She is the director of Interventional Cardiology department in Germans Trias i Pujo University Hospital in Badalona (Barcelona). The main topics of his scientific work are coronary imaging (mainly intravascular ultrasound). She is a member of European cardiology society and previous president of Spanish Interventional cardiology society.

Xavier Carrillo, MD., is an invasive cardiologist graduated in Medicine in 2003 at University of Barcelona. He works in Germans Trias i Pujol University Hospital in Badalona (Barcelona). The main topics of his scientific work are Acute Coronary Syndromes and intravascular coronary imaging. He is a member of European and Spanish cardiology societies. He is a member of AIM code committee at Catalan department of health.

Tomas Kovarnik, MD., Ph.D. is an invasive cardiologist graduated in 1998 at Charles University, with PhD defense 2012. He works in Charles University Hospital in Prague. The main topics of his scientific work are coronary imaging (mainly intravascular ultrasound) and development of coronary atherosclerosis. He is a member of European and Czech cardiology societies.

Ching-Wei Wang is the director of Biomedical Image and Computer Vision Lab in the Graduate Institute of Biomedical Engineering at the National Taiwan University of Science and Technology. Ching-Wei has years of working experiences in computer vision and machine learning, and her current interests include medical image registration, image segmentation and pattern recognition.

Hsiang-chou Chen conducted his studies on medical image in Prof Ching-Wei Wang's Biomedical Image and Computer Vision group at the National Taiwan University of Science and Technology from 2011 to 2013

Themis P. Exarchos was born in Ioannina, Greece, in 1980. He received the Diploma degree in computer engineering and informatics from the University of Patras, Patras, Greece, in 2003 and the PhD degree from the Dept. of Medical Physics of the University of Ioannina. His PhD thesis is entitled Data Mining and Healthcare decision support systems. He is with the Foundation for Research and Technology, Institute of Molecular Biology and Biotechnology, Dept of Biomedical Research Institute. Dr Exarchos has worked in several R\&D projects and has published more that 100 papers in scientific journals, conferences and books.

Dimitrios I. Fotiadis received the Diploma degree in chemical engineering from the National Technical University of Athens, in 1985, and the Ph.D. degree in chemical engineering and materials science from the University of Minnesota in 1990. He is a Professor of Biomedical Engineering in the Department of Materials Science and Engineering, University of Ioannina. He was a Visiting Researcher at the RWTH Aachen, Germany, and the Massachusetts Institute of Technology, Boston. He has coordinated and participated in several R\&D funded projects. He is the author or coauthor of more than 150 papers in scientific journals, 300 papers in peer-reviewed conference proceedings, and more than 30 chapters in books. He is also the editor or coeditor of 16 books.

François Destrempes received the BSc degree in mathematics from the Universite de Montréal (1985) and the MSc (1987) and PhD (1990) degrees in mathematics from Cornell University. He was a postdoctoral fellow at the Centre de Recherche Mathématiques of the Université de Montréal (1990-1992). He received a postgraduate degree (2000) in applied computer science and the MSc (2002), and PhD (2006) degrees in computer science from the Université de Montréal. He was a postdoctoral fellow at the Laboratory of Biorheology and Medical Ultrasonics at the Research Center of the University of Montreal Hospital, where is currently a research associate.

Guy Cloutier is Director of the Laboratory of Biorheology and Medical Ultrasonics at the University of Montreal Hospital Research Center (www.lbum-crchum.com) Professor and Director of Research at the Department of Radiology, Radio-Oncology and Nuclear Medicine at the University of Montreal, and Member of the Institute of Biomedical Engineering at the same university. His research interests are in quantitative ultrasound imaging of red blood cell aggregation; quasi-static and dynamic ultrasound elastography of atherosclerotic plaques, vascular aneurysms, 
deep vein thrombi and breast cancers; ultrasound image analysis; 3-D morphologic and hemodynamic assessment of lower-limb arterial stenoses; and mathematical and biomechanical modeling. He has published more than 140 peer-reviewed articles in these fields, holds 12 patents, licensed two technologies, and was recipient of the National Scientist award of the Fonds de la Recherche en Santé du Québec (2004-2009).

Oriol Pujol Vila is associate professor at Dept. of Applied Mathematics and Analysis at the University of Barcelona, and senior researcher at the Computer Vision Center. His main research areas are machine learning and computer vision. In general, he is interested in the development and application of machine learning techniques to visual object recognition and wearable sensors in the eHealth domain. In applied research he has worked in IVUS image analysis, human behavior analysis and egocentric wearable computing for mixed and augmented reality.

Marina Alberti received her BSc and MSc degrees in biomedical engineering from University of Genova, Italy, in 2007 and 2009, respectively. She received her PhD degree in applied mathematics from University of Barcelona and Computer Vision Center (CVC), Spain, in 2013. Presently, she is a postdoctoral researcher at the Computer Vision and Active Perception Lab of the Royal Institute of Technology (KTH), Stockholm, Sweden. Her research interests include medical imaging analysis, computer vision and machine learning.

E. Gerardo Mendizabal received the B.E. degree in electronics from the Instituto Tecnologico y de Estudios Superiores de Occidente, Guadalajara, México, in 2003, the M. Sc. degree in computer science and industrial mathematics from the Center for Research in Mathematics (CIMAT), Guanajuato, México in 2007, and the Ph. D. degree in computer science from the University of Houston, U.S.A. in 2012. He is currently a research scientist at the University of Guadalajara, México. His current research interest include computer vision, medical image processing, DNA analysis and artificial life.
Mariano Rivera received the B.E. degree in electronics from the Durango Institute of Technology, Durango, Mexico, in 1989, the M.Sc. degree in electronics from the Chihuahua Institute of Technology, Chihuahua, Mexico, in 1993, and the D.Sc. degree in optics from the Center for Research in Optics, Leon, Mexico, in 1997. Since 1997, he has been with the Department of Computer Science, Center for Research in Mathematics (CIMAT), Guanajuato, Mexico. His research is summarized in more than 80 papers in scientific journals and conference proceeding papers. His current research interests include computer vision, medical image processing, machine learning, and optimization. Dr. Rivera is a Fellow of the National Researcher System (SNI) of the Mexican Government

Timur Aksoy has received his BA from University of Pennsylvania and MS from Izmir University of Economics. Since 2009, he continues PhD studies in Computer Science \& Engineering program at Sabanci University. Currently, his research subject is coronary artery registration in the field of Medical Image Processing.

Richard Downe completed his BS in electrical engineering at Trinity College in Hartford, CT in 2003, and his doctorate in the same at the University of Iowa in 2013, doing his research in the predictive analysis of the progression of cardiovascular disease based upon radiological, demographic, and serum biomarker data. He currently lives in San Jose, CA and works at IBM's Almaden Research Center.

Ioannis A. Kakadiaris is Hugh Roy and Lillie Cranz Cullen University Professor of Computer Science, Electrical \& Computer Engineering, and Biomedical Engineering at UH. His research interests include computer vision, pattern recognition, biomedical image analysis, and biometrics. Dr. Kakadiaris is the recipient of a number of awards, including the NSF Early Career Development Award, Schlumberger Technical Foundation Award, UH Computer Science Research Excellence Award, UH Enron Teaching Excellence Award, and the James Muller Vulnerable Plaque Young Investigator Prize. His research has been featured on The Discovery Channel, National Public Radio, KPRC NBC News, KTRH ABC News, and KHOU CBS News. 